\title{
Multiplicative Consistency of Intuitionistic Reciprocal Preference Relations and Its Application to Missing Values Estimation and Consensus Building
}

\author{
Jian $\mathrm{Wu}^{\mathrm{a}, \mathrm{b}}$, Francisco Chiclana ${ }^{\mathrm{b}}$ \\ ${ }^{a}$ School of Economics and Management, Zhejiang Normal University, Jinhua, Zhejiang, China \\ ${ }^{b}$ Centre for Computational Intelligence, Faculty of Technology, De Montfort University, Leicester, UK
}

\section{Abstract}

The mathematical modelling and representation of Tanino's multiplicative transitivity property to the case of intuitionistic reciprocal preference relations (IRPRs) is derived via Zadeh's extension principle and the representation theorem of fuzzy sets. This result guarantees the correct generalisation of the multiplicative transitivity property of reciprocal preference relations (RPRs), and it allows the multiplicative consistency (MC) property of IRPRs to be defined. The MC property used in decision making problems is threefold: (1) to develop a consistency based procedure to estimate missing values in IRPRs using an indirect chain of alternatives; (2) to quantify the consistency index (CI) of preferences provided by experts; and (3) to build a novel consistency based induced ordered weighted averaging (MC-IOWA) operator that associates a higher contribution in the aggregated value to the more consistent information. These three uses are implemented in developing a consensus model for GDM problems with incomplete IRPRs in which the level of agreement between the experts' individual IRPRs and the collective IRPR, which is referred here as the proximity index (PI), is combined with the CI to design a feedback mechanism to support experts to change some of their preference values using simple advice rules that aim at increasing the level of agreement while, at the same time, keeping a high degree of consistency. In the presence of missing information, the feedback mechanism implements the consistency based procedure to produce appropriate estimate values of the missing ones based on the given information provided by the experts. Under the assumption of constant CI values, the feedback mechanism is proved to converge to unanimous consensus when all experts are provided with recommendations and these are fully implemented. Additionally, visual representation of experts' consensus position within the group before and after implementing their feedback advice is also provided, which help an expert to revisit his evaluations and make changes if considered appropriate to achieve a higher consensus level. Finally, an IRPR fuzzy majority based quantifier-guided nondominance degree based prioritisation method using the associated score reciprocal preference relation is proposed to obtain the final solution of consensus.

\footnotetext{
${ }^{2}$ Cite as: Jian Wu, Francisco Chiclana: Multiplicative consistency of intuitionistic reciprocal preference relations and its application to missing values estimation and consensus building. Knowledge-Based Systems Volume 71, November 2014, pages 187200. doi: 10.1016/j.knosys.2014.07.024

Email addresses: jyajian@163.com (Jian Wu), chiclana@dmu.ac.uk (Francisco Chiclana)
} 
Keywords: Multiplicative consistency, Intuitionistic reciprocal preference relations, Missing preferences, Group decision and negotiation, Consensus

\section{Introduction}

Group decision making (GDM) consists of multiple individuals interacting to reach a decision based on the information they provide. Given two alternatives of a finite set of all potentially available, $X$, an expert either prefers one to the other or is indifferent between them. Obviously, there is another possibility, that of an expert being unable to compare them.

There exist two main mathematical frameworks based on the concept of preference relation. In the first one a preference relation is defined for each one of the above three possible preference states, which is usually referred to as a preference structure on the set of alternatives [33]. The second one integrates the three possible preference states into a single preference relation [5]. Further to this, in each case two different representations could be adopted: the use of binary (crisp) preference relations or the use of $[0,1]$-valued (fuzzy) preference relations. Reciprocal $[0,1]$-valued relations $\left(P=\left(p_{i j}\right) ; \forall i, j: 0 \leq p_{i j} \leq 1, p_{i j}+p_{j i}=1\right)$ are frequently used in fuzzy set theory for representing intensities of preferences $[5,10,36]$. These types of relations will be referred to as simply reciprocal preference relations, and are the ones used in this paper. In probabilistic choice theory, reciprocal preference relations describe the binary preferences subsets of two-alternatives of $X$, and are known with the name 'probabilistic binary preference relations' [30]. Reciprocal preference relations can be seen as a particular case of (weakly) complete fuzzy preference relations [22], i.e. fuzzy preference relations satisfying $p_{i j}+p_{j i} \geq 1 \forall i, j$.

An exhaustive survey of the second type of preference relations mentioned above is given in [43], with the main relations used in the literature to capture uncertainty of information being: the multiplicative preference relation (MPR), the reciprocal preference relation (RPR), the linguistic preference relation (LPR), and the intuitionistic reciprocal preference relation (IRPR). The last one is based on Atanassov's intuitionistic fuzzy set (IFS) introduced in [3]. An IFS is based on the use of membership degree, non-membership degree and hesitation index to model experts' subjective preferences. Indeed, there may be some real-life decision making cases where a decision maker (DM) may not be able to accurately express his/her preferences for some or all of the alternatives because he/she is not completely confident or presents some hesitation [17], making they use of intuitionistic fuzzy values very suitable to model and represent the DM's preference rather than other type of preference representation formats [44]. Intuitionistic fuzzy values have been used for example in Iwate (Japan) by Professor Fujita and his research laboratory to represent and model medical doctor responses in medical diagnosis as part of the mental cloning "used to mirror a person cognitive behaviour into a model that interacts with human users" [6] on building the virtual doctor system (VDS) for medical applications [7-9]. Recently, the use of IRPRs in decision making in uncertain environments has 
attracted the attention of many researchers [23, 24, 39].

An issue to address in GDM problems with IRPRs is the lack of information, a problem extensively studied in the case of RPRs $[14,20]$. In this context, consistency based methods to 'estimate' the missing values from known ones have been proposed in $[1,2,27]$, which later were extended to the GDM framework [28, 29]. However, modelling and evaluating the consistency of IRPRs has not yet been solved.

'Some individual opinions can be considered more consistent than other individual opinions', which was used by Cutello and Montero in [16] to claim that rationality of individuals can be considered a fuzzy concept, where they addressed the problem of modelling rationality of individuals based only on their opinions over a finite and fixed set of alternatives expressed using complete fuzzy preference relations. They characterised fuzzy rationality measures which are explicitly consistent by establishing a collection of conditions to satisfy. Explicit consistency is defined in [16] as 'absence of explicit contradictions, i.e. statements of type $(P \wedge \neg P)$.'

Consistency of RPRs has been modelled using the notion of transitivity, in the sense that if alternative $x_{i}$ is preferred to alternative $x_{j}\left(p_{i j} \geq 0.5\right)$ and this one to $x_{k}\left(p_{j k} \geq 0.5\right)$ then alternative $x_{i}$ should be preferred to $x_{k}\left(p_{i k} \geq 0.5\right)$. This transitivity notion is normally referred to as weak stochastic transitivity [30, 36]. However, the implementation of the intensity of preference in modelling consistency of RPRs has been proposed in many different ways [27]. Tanino in [36] proposed the additive transitivity property, which is although equivalent to Saaty's consistency property for MPRs [35], is in conflict with the $[0,1]$ scale used for providing the preference values and therefore it is an inappropriate property to model consistency of reciprocal preference relations [12]. In [36], Tanino also proposed a multiplicative transitivity property for RPRs, which has been characterised to be the most appropriate one for modelling cardinal consistency of RPRs [12]. Recall that RPRs are particular cases of IRPRs, and therefore the same previous claim can be applied to them. Thus, the first objective in this paper is to formalise the multiplicative transitivity property for IRPRs. Once this is achieved, a methodology will be developed to (1) quantify the level of consistency or consistency index (CI) of an IRPR, and (2) estimate missing values of incomplete IRPRs. Because consistent information is considered more relevant or important than inconsistent information, an intuitionistic aggregation operator that associates higher weights with more consistent information will be developed. In other words, a new MC induced ordered weighted averaging (MC-IOWA) operator is defined and proposed to compute the collective IRPR.

As aforementioned, consistency is linked to rationality of individuals, and therefore it has been considered as a reasonable criteria to guide consensus reaching processes [11,37]. On the other hand, similarity interpreted as a measure of general or widespread agreement, based on the use of a metric or distance function, is usually regarded as a criteria to use in measuring consensus $[15,18,40,50]$. By combining both consistency and similarity functions, Herrera-Viedma et al. [29] developed a feedback 
mechanism to provide advice to experts in order to increase the consensus level of the group. Chiclana et al. [13] and $\mathrm{Wu}$ and $\mathrm{Xu}$ [42] designed a two stage model with a first stage aiming to reach acceptable consistency level while the second one was used to achieve a predefined consensus level. Different from the above consensus models, Dong et al. [19] investigated a minimum cost optimization model to reach acceptable consensus in which the individual consistency and consensus level are used as two limiting conditions simultaneously. Obviously, using these two criteria simultaneously in consensus process seems to be more reliable than just one criteria.

Therefore, the second objective of this paper is to investigate a consensus reaching process for IRPRs that combines these two criteria. Approaches to model consensus in GDM problems with IRPRs are already available in literature [34, 44]. However, there exist two main limitations in these consensus models: (i) they did not take into account of the consistency of individual preference; and (ii) they are static in nature because, when there is not enough consensus, they do not include any type of feedback process to advise the experts on how to change their preferences to increase consensus. To overcome these limitations, and inspired by the work of Herrera-Viedma et al. [29], both consistency and consensus levels will be implemented in the design of a feedback mechanism to support experts to change some of their preference values using simple advice rules that aim at increasing the level of agreement while, at the same time, keeping a high degree of consistency. In the presence of missing information, the feedback mechanism implements the consistency based procedure to produce appropriate estimate values of the missing ones based on the given information provided by the experts. Under the assumption of constant CI values, the feedback mechanism is proved to converge to unanimous consensus when all experts are provided with recommendations and these are fully implemented. Additionally, visual representation of experts' consensus position within the group before and after implementing their feedback advice is also provided, which helps an expert to revisit his evaluations and make changes if considered appropriate to achieve a higher consensus level. Finally, an IRPR fuzzy majority based quantifier-guided non-dominance degree based prioritisation method using the associated score reciprocal preference relation is proposed to obtain the final solution of consensus.

The rest of paper is set out as follows: Section 2 presents the formal approach to extend the mathematical expression of multiplicative transitivity property from RPR to IRPR, as well as the definition of multiplicative consistent IRPR and consistency indexes of IRPRs. In Section 3, a multiplicative consistency based method to estimate missing values of IRPRs is proposed. The consensus model for GDM with incomplete IRPRs is covered in Section 4, with special attention paid to the design of the consistency-consensus based feedback mechanism. To illustrate the complete application of the multiplicative consistency based consensus model for GDM with incomplete IRPRs, an example is given in Section 5. Finally, Section 6 gives an analysis of the proposed consensus model highlighting the main differences with respect to existing consensus models in the literature, and then draws the 
conclusions.

\section{Consistency of Intuitionistic Reciprocal Preference Relations}

The concept of an Intuitionistic Fuzzy Set (IFS) was introduced by Atanassov in [3]:

Definition 1 (Intuitionistic fuzzy set (IFS)). An intuitionistic fuzzy set (IFS) $A$ over a universe of discourse $X$ is represented as $A=\left\langle\mu_{A}, \nu_{A}\right\rangle$ where $\mu_{A}: X \rightarrow[0,1], \nu_{A}: X \rightarrow[0,1]$ and $0 \leq \mu_{A}(x)+$ $\nu_{A}(x) \leq 1 \forall x \in X$. For each $x \in X$, the numbers $\mu_{A}(x)$ and $\nu_{A}(x)$ are known as the degree of membership and degree of non-membership of $x$ to $A$, respectively.

An IFS becomes a FS when $\mu_{A}(x)=1-\nu_{A}(x) \forall x \in X$. However, when there exists at least a value $x \in X$ such that $\mu_{A}(x)<1-\nu_{A}(x)$, an extra parameter has to be taken into account when working with IFSs: the hesitancy degree, $\tau_{A}(x)=1-\mu_{A}(x)-\nu_{A}(x)$, which represents the amount of lacking information in determining the membership of $x$ to $A$. If the hesitation degree is zero, the reciprocal relationship between membership and non-membership makes the latter one unnecessary in the formulation as it can be derived from the former.

\subsection{Intuitionistic Reciprocal Preference Relation}

It is well known that when we have three alternatives $x_{i}, x_{j}, x_{k}$ such that $x_{i}$ is preferred to $x_{j}$ and $x_{j}$ to $x_{k}$, the question whether the 'degree or strength of preference' of $x_{i}$ over $x_{j}$ exceeds, equals, or is less than the 'degree or strength of preference' of $x_{j}$ over $x_{k}$ cannot be answered using binary (crisp) preference relations [12]. A solution to this issue requires an appropriate representation of intensity of preference between alternatives. This can be achieved by implementing the concept of fuzzy set in preference modelling, which when applied to a binary relation leads to the concept of a fuzzy relation. In [5], we can find for the first time the concept of a reciprocal (fuzzy) preference relation that integrates the three possible preference states into a single preference relation. The adapted definition of a RPR is the following:

Definition 2 (Reciprocal Preference Relation (RPR)). A RPR P on a finite set of alternatives $X=\left\{x_{1}, \ldots, x_{n}\right\}$ is characterised by a membership function $\mu_{P}: X \times X \longrightarrow[0,1], \mu_{P}\left(x_{i}, x_{j}\right)=p_{i j}$, verifying $p_{i j}+p_{j i}=1 \forall i, j \in\{1, \ldots, n\}$.

A RPR may be conveniently denoted by the matrix $P=\left(p_{i j}\right)$, with the following interpretation: $p_{i j}=0.5$ indicates indifference between $x_{i}$ and $x_{j} ; p_{i j} \in(0.5,1]$ indicates a definite preference for $x_{i}$ over $x_{j}$, and $p_{i j}=1$ indicates the maximum degree of preference for $x_{i}$ over $x_{j}$. In [44], Xu defined the intuitionistic RPR (IRPR) as a generalisation of the concept of RPR as follows: 
Definition 3 (Intuitionistic RPR (IRPR)). An IRPR $R$ on a finite set of alternatives $X$ is characterised by a membership function $\mu_{R}: X \times X \rightarrow[0,1]$ and a non-membership function $\nu_{R}: X \times X \rightarrow$ $[0,1]$ such that $0 \leq \mu_{R}\left(x_{i}, x_{j}\right)+\nu_{R}\left(x_{i}, x_{j}\right) \leq 1 \quad \forall\left(x_{i}, x_{j}\right) \in X \times X$. The value $\mu_{R}\left(x_{i}, x_{j}\right)=\mu_{i j}$ is interpreted as the certainty degree up to which $x_{i}$ is preferred to $x_{j}$, while the value $\nu_{R}\left(x_{i}, x_{j}\right)=\nu_{i j}$ represents the certainty degree up to which $x_{i}$ is non-preferred to $x_{j}$. Additionally, the following conditions are imposed:

$$
\mu_{i i}=\nu_{i i}=0.5 \forall i \in\{1, \ldots, n\} ; \quad \mu_{j i}=\nu_{i j} \forall i, j \in\{1, \ldots, n\}
$$

Using matrix notation, an IRPR is represented as $R=\left(r_{i j}\right)=\left(\left\langle\mu_{i j}, \nu_{i j}\right\rangle\right)$. When the hesitancy degree function is the null function then $\mu_{i j}+\nu_{i j}=1 \forall i, j$, and therefore the IRPR $R=\left(r_{i j}\right)=$ $\left(\left\langle\mu_{i j}, \nu_{i j}\right\rangle\right)$ is mathematically equivalent to the $\operatorname{RPR} P=\left(p_{i j}\right)=\left(\mu_{i j}\right)$. Some examples of the use of IRPRs in the context of public relation and mass communication are discussed in [4].

\subsection{Multiplicative Transitivity Property of an IRPR}

Rationality is related with consistency [16], which is associated with the transitivity property [27]. For RPRs many properties have been suggested to model transitivity, some of which have been proved to be inappropriate in [12]. The assumption of experts being able to quantify their preferences in the domain $[0,1]$, instead of $\{0,1\}$, underlies unlimited computational abilities and resources from the experts, which was used by Chiclana et al. in [12] to propose the modelling of the cardinal consistency of RPRs via a functional equation, and they proved that when such a function is almost continuous and monotonic (increasing) then it must be a representable uninorm. Cardinal consistency with the conjunctive representable Cross Ratio uninorm is equivalent to Tanino's multiplicative transitivity property (more details given later in Section 3). Because any two representable uninorms are order isomorphic, then multiplicative transitivity is indeed characterised as the most appropriate to model consistency of RPRs. Obviously, multiplicative transitivity property extends weak stochastic transitivity, and therefore extends the classical transitivity property of crisp preference relations. The following definition summarises this result:

Definition 4 (Multiplicative Transitivity Property of RPR). A RPR $P=\left(p_{i j}\right)$ on a finite set of alternatives $X$ is multiplicative transitive if and only if

$$
p_{i j} \cdot p_{j k} \cdot p_{k i}=p_{i k} \cdot p_{k j} \cdot p_{j i} \quad \forall i, k, j \in\{1,2, \ldots n\}
$$

Multiplicative transitivity property was proposed by Tanino when $p_{i j}>0 \forall i, j[36]$. For IRPR, $\mathrm{Xu}$ proposed the following definition of the multiplicative transitivity property [44]

$$
r_{i j}=r_{i k} \cdot r_{k j} \forall i, j, k=1,2, \ldots n
$$

where $r_{i k} \cdot r_{k j}=\left\langle\mu_{i k} \cdot \mu_{k j}, \nu_{i k}+\nu_{k j}-\nu_{i k} \cdot \nu_{k j}\right\rangle$ is the product operation of IFSs as defined by Atanassov in $[3]$. 
However, it is quite straight forward to conclude that property (2) is in contradiction with the multiplicative transitivity of RPRs and, consequently, with classical transitivity of crisp preferences. Indeed, when the hesitation degree is zero the IRPR is a RPR and therefore for property (2) to be appropriate to model transitivity should also extend weak stochastic transitivity. However, this is clearly not the case: the following values $p_{i k}=0.6\left(x_{i} \succ x_{k}\right)$ and $p_{k j}=0.7\left(x_{k} \succ x_{j}\right)$ would result, using property (2), in a value of $p_{i j}=0.42<0.5\left(x_{j} \succ x_{i}\right)$.

In what follows, we will formally generalise the multiplicative transitivity property for RPRs to IRPRs. We will do this by applying Zadeh's Extension Principle [52] to the case when the preference values are fuzzy sets rather than crisp values in [0,1]. The Representation Theorem [51] will be applied to the corresponding fuzzy sets that are obtained after applying the extension principle, so that we can obtain the corresponding multiplicative transitivity property of fuzzy preference values. Next, we apply this result to the case when the fuzzy sets are interval-valued fuzzy sets, i.e. fuzzy sets whose membership function is always 1 for all values in a closed interval and zero otherwise. This process will allow us to extend in a correct way the multiplicative transitivity property from RPRs to interval-valued RPRs (IVRPRs). Finally, we will use the mathematical isomorphism between IFSs and IVFSs [17] to obtain the correct formulation of the multiplicative transitivity property for IRPRs.

The extension principle allows the domain of a functional mapping to be extended from crisp elements to fuzzy sets as given below [25]:

Definition 5 (Extension Principle). Let $X_{1} \times X_{2} \times \ldots \times X_{n}$ be a universal product set and $F$ a functional mapping of the form

$$
F: X_{1} \times X_{2} \times \ldots \times X_{n} \longrightarrow Y
$$

that maps the element $\left(x_{1}, x_{2}, \ldots, x_{n}\right) \in X_{1} \times X_{2} \times \ldots \times X_{n}$ to the element $y=F\left(x_{1}, x_{2}, \ldots, x_{n}\right)$ of the universal set $Y$. Let $A_{i}$ be a fuzzy set over the universal set $X_{i}$ with membership function $\mu_{A_{i}}(i=1,2, \ldots, n)$. The membership function $\mu_{B}$ of the fuzzy set $B=F\left(A_{1}, \ldots, A_{n}\right)$ over the universal set $Y$ is

$$
\mu_{B}(y)= \begin{cases}\sup _{y=F\left(x_{1}, x_{2}, \ldots, x_{n}\right)}\left[\mu_{A_{1}}\left(x_{1}\right) * \mu_{A_{2}}\left(x_{2}\right) * \ldots * \mu_{A_{n}}\left(x_{n}\right)\right] & \text { if } \exists x_{1}, \ldots, x_{n}: y=F\left(x_{1}, \ldots, x_{n}\right) \\ 0 & \text { otherwise }\end{cases}
$$

where $*$ is a t-norm.

For the work presented in this paper, the minimum t-norm $(\wedge)$ is used. Expression $(1)$ involves the comparison of two products of three crisp numbers (preference values) in the unit interval $[0,1]$. In what follows we will extend the function $f:[0,1] \times[0,1] \times[0,1] \longrightarrow[0,1]$,

$$
f\left(x_{1}, x_{2}, x_{3}\right)=x_{1} \cdot x_{2} \cdot x_{3},
$$


to $f\left(A_{1}, A_{2}, A_{3}\right)$ where $A_{1}, A_{2}, A_{3}$ are fuzzy sets over the set $[0,1]$ and associated membership functions $\mu_{A_{1}}, \mu_{A_{2}}, \mu_{A_{3}}$, respectively. The extension principle states that $B=f\left(A_{1}, A_{2}, A_{3}\right)$ is a fuzzy set over the set $[0,1]$ with membership function $\mu_{B}:[0,1] \rightarrow[0,1]$;

$$
\mu_{B}(y)=\sup _{\substack{x_{1} \cdot x_{2} \cdot x_{3}=y \\ x_{1}, x_{2}, x_{3} \in[0,1]}}\left[\mu_{A_{1}}\left(x_{1}\right) \wedge \mu_{A_{2}}\left(x_{2}\right) \wedge \mu_{A_{3}}\left(x_{3}\right)\right]
$$

The representation theorem of fuzzy sets provides an alternative and convenient way to define a fuzzy set via its corresponding family of crisp $\alpha$-level sets. The $\alpha$-level set of a fuzzy set $A$ over the universe $Z$ is defined as $A^{\alpha}=\left\{z \in Z \mid \mu_{A}(z) \geq \alpha\right\}$. The set of crisp sets $\left\{A^{\alpha} \mid 0<\alpha \leq 1\right\}$ is said to be a representation of the fuzzy set $A$. Indeed, the fuzzy set $A$ can be represented as

$$
A=\underset{0<\alpha \leq 1}{\cup} \alpha A^{\alpha}
$$

with membership function

$$
\mu_{A}(z)=\sup _{\alpha: z \in A_{\alpha}} \alpha
$$

Let $A_{1}^{\alpha}, A_{2}^{\alpha}$ and $A_{3}^{\alpha}$ be the $\alpha$-level sets of fuzzy sets $A_{1}, A_{2}$ and $A_{3}$ described above. We have

$$
f\left(A_{1}^{\alpha}, A_{2}^{\alpha}, A_{3}^{\alpha}\right)=\left\{x_{1} \cdot x_{2} \cdot x_{3} \mid x_{1} \in A_{1}^{\alpha}, x_{2} \in A_{2}^{\alpha}, x_{3} \in A_{3}^{\alpha}\right\} .
$$

Both $B^{\alpha}$ and $f\left(A_{1}^{\alpha}, A_{2}^{\alpha}, A_{3}^{\alpha}\right)$ are crisp sets. Furthermore, as we prove next, we have the following equality:

$$
B^{\alpha}=f\left(A_{1}^{\alpha}, A_{2}^{\alpha}, A_{3}^{\alpha}\right)
$$

I. Let $y \in B^{\alpha}$. By definition, we have $\mu_{B}(y) \geq \alpha$ and there exists at least three values $x_{1}, x_{2}, x_{3} \in$ $[0,1]$ such that $x_{1} \cdot x_{2} \cdot x_{3}=y$ and $\left[\mu_{A_{1}}\left(x_{1}\right) \wedge \mu_{A_{2}}\left(x_{2}\right) \wedge \mu_{A_{3}}\left(x_{3}\right)\right] \geq \alpha$. Therefore, $\mathrm{t} \mu_{A_{1}}\left(x_{1}\right) \geq \alpha$, $\mu_{A_{2}}\left(x_{2}\right) \geq \alpha$ and $\mu_{A_{3}}\left(x_{3}\right) \geq \alpha$, which means that $x_{1} \in A_{1}^{\alpha}, x_{2} \in A_{2}^{\alpha}$ and $x_{3} \in A_{3}^{\alpha}$. Consequently, $y \in f\left(A_{1}^{\alpha}, A_{2}^{\alpha}, A_{3}^{\alpha}\right)$, i.e. $B^{\alpha} \subseteq f\left(A_{1}^{\alpha}, A_{2}^{\alpha}, A_{3}^{\alpha}\right)$.

II. Let $y \in f\left(A_{1}^{\alpha}, A_{2}^{\alpha}, A_{3}^{\alpha}\right)$. There exist $x_{1} \in A_{1}^{\alpha}, x_{2} \in A_{2}^{\alpha}$ and $x_{3} \in A_{3}^{\alpha}$ such that $x_{1} \cdot x_{2} \cdot x_{3}=y$. We have that $\mu_{A_{1}}\left(x_{1}\right) \geq \alpha, \mu_{A_{2}}\left(x_{2}\right) \geq \alpha$ and $\mu_{A_{3}}\left(x_{3}\right) \geq \alpha$ and therefore:

$$
\sup _{\substack{x_{1} \cdot x_{2} \cdot x_{3}=y \\ x_{1} \in A_{1}^{\alpha}, x_{2} \in A_{2}^{\alpha}, x_{3} \in A_{3}^{\alpha}}}\left[\mu_{A_{1}}\left(x_{1}\right) \wedge \mu_{A_{2}}\left(x_{2}\right) \wedge \mu_{A_{3}}\left(x_{3}\right)\right] \geq \alpha .
$$

Because $A_{1}^{\alpha}, A_{2}^{\alpha}, A_{3}^{\alpha} \subseteq[0,1]$, then we have:

$$
\sup _{\substack{x_{1} \cdot x_{2} \cdot x_{3}=y \\ x_{1}, x_{2}, x_{3} \in[0,1]}}\left[\mu_{A_{1}}\left(x_{1}\right) \wedge \mu_{A_{2}}\left(x_{2}\right) \wedge \mu_{A_{3}}\left(x_{3}\right)\right] \geq \sup _{\substack{x_{1} \cdot x_{2} \cdot x_{3}=y \\ x_{1} \in A_{1}^{\alpha}, x_{2} \in A_{2}^{\alpha}, x_{3} \in A_{3}^{\alpha}}}\left[\mu_{A_{1}}\left(x_{1}\right) \wedge \mu_{A_{2}}\left(x_{2}\right) \wedge \mu_{A_{3}}\left(x_{3}\right)\right] .
$$

We conclude that $y \in B^{\alpha}$, i.e. $f\left(A_{1}^{\alpha}, A_{2}^{\alpha}, A_{3}^{\alpha}\right) \subseteq B^{\alpha}$.

An example of the multiplication of fuzzy numbers using the extension principle and the representation theorem is shown below. Figure 1 shows three fuzzy numbers $A_{1}, A_{2}$ and $A_{3}$ with trapezoidal 
membership functions, which could be used to represent concepts such as 'equally preferred', 'slightly preferred to' and 'highly preferred to' $[26,32]$. The fuzzy set $A_{1} \cdot A_{2} \cdot A_{3}$ is constructed by applying Eq. (4) to compute the lower and upper bounds of its $\alpha$-level sets, followed by the application of the representation theorem of fuzzy sets. The computation of the lower bound of the $0.2-$ level set of $A_{1} \cdot A_{2} \cdot A_{3}$ is also shown in Fig. 1.

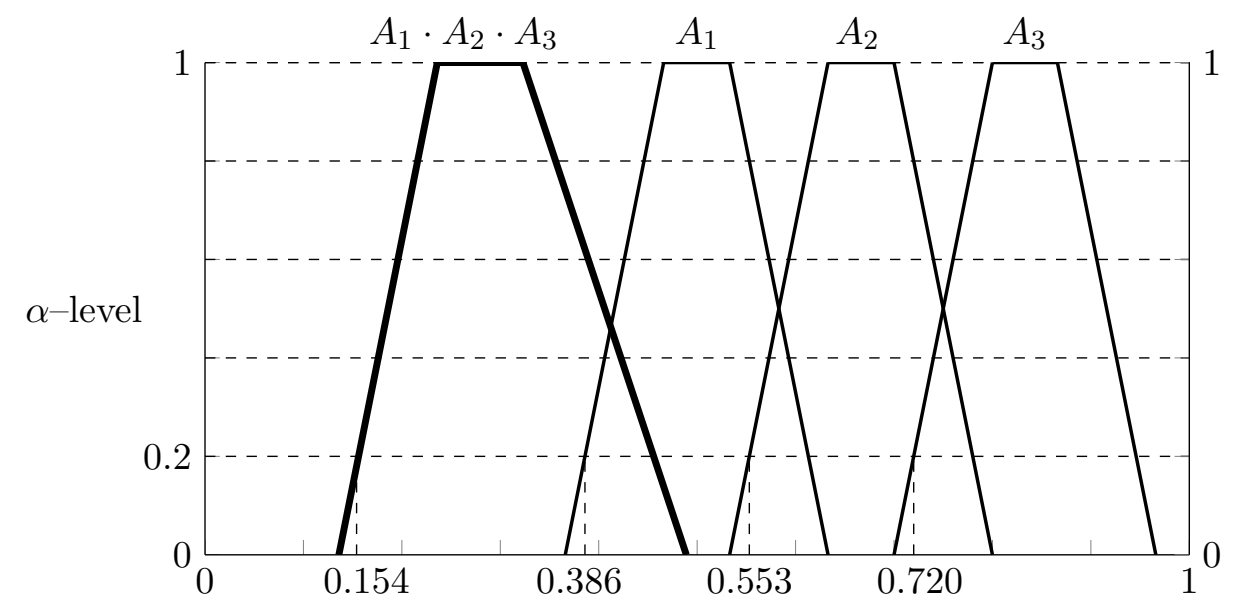

Figure 1: $\alpha$-level multiplication of three fuzzy numbers with trapezoidal membership functions

When $A_{1}, A_{2}$ and $A_{3}$ are closed intervals their membership function are

$$
\mu_{A_{1}}\left(x_{1}\right)=\left\{\begin{array}{ll}
1 & x_{1} \in\left[a^{-}, a^{+}\right] \\
0 & \text { Otherwise }
\end{array} ; \mu_{A_{2}}\left(x_{2}\right)=\left\{\begin{array}{ll}
1 & x_{2} \in\left[b^{-}, b^{+}\right] \\
0 & \text { Otherwise }
\end{array} ; \mu_{A_{3}}\left(x_{3}\right)=\left\{\begin{array}{ll}
1 & x_{3} \in\left[c^{-}, c^{+}\right] \\
0 & \text { Otherwise }
\end{array} ;\right.\right.\right.
$$

and they have associated only one non-empty $\alpha$-level set, the 1-level set. On the one hand, applying (4), $f\left(A_{1}, A_{2}, A_{3}\right)=f\left(A_{1}^{1}, A_{2}^{1}, A_{3}^{1}\right)$. On the other hand, interval arithmetic yields:

$$
f\left(A_{1}^{1}, A_{2}^{1}, A_{3}^{1}\right)=\left[a^{-} \cdot b^{-} \cdot c^{-}, a^{+} \cdot b^{+} \cdot c^{+}\right]
$$

This result justifies the following definitions of multiplicative transitivity property of IVRPR and IRPR, respectively. Let $\tilde{P}([0,1])$ be the set of closed intervals in $[0,1]$. An IVRPR is defined as follows:

Definition 6 (Interval-valued RPR(IVRPR)). An IVRPR $B$ on a finite set of alternatives $X=$ $\left\{x_{1}, \ldots, x_{n}\right\}$ is characterised by a membership function $\mu_{B}: X \times X \longrightarrow \tilde{P}([0,1])$ with $\mu_{B}\left(x_{i}, x_{j}\right)=$ $\left[b_{i j}^{-}, b_{i j}^{+}\right]$verifying $b_{i j}^{-}+b_{j i}^{+}=b_{i j}^{+}+b_{j i}^{-}=1, b_{i i}^{+}=b_{i i}^{-}=0.5 \quad \forall i, j=1,2, \ldots n$.

In the following, we state the multiplicative transitivity property for IVRPRs:

Definition 7 (Multiplicative Transitivity Property of IVRPR). An IVRPR $B=\left(b_{i j}\right)=\left(\left[b_{i j}^{-}, b_{i j}^{+}\right]\right)$ is multiplicative transitive if and only if

$$
\forall i, j, k:\left\{\begin{array}{l}
b_{i j}^{-} \cdot b_{j k}^{-} \cdot b_{k i}^{-}=b_{i k}^{-} \cdot b_{k j}^{-} \cdot b_{j i}^{-} \\
b_{i j}^{+} \cdot b_{j k}^{+} \cdot b_{k i}^{+}=b_{i k}^{+} \cdot b_{k j}^{+} \cdot b_{j i}^{+}
\end{array}\right.
$$


Because the IRPR $R=\left(r_{i j}\right)=\left(\left\langle\mu_{i j}, \nu_{i j}\right\rangle\right)$ is isomorphic to the IVRPR $B=\left(b_{i j}\right)=\left(\left[\mu_{i j}, 1-\nu_{i j}\right]\right)$, the multiplicative transitivity property of IRPRs can be defined as follows:

Definition 8 (Multiplicative Transitivity Property of IRPR). An IRPR $R=\left(r_{i j}\right)=\left(\left\langle\mu_{i j}, \nu_{i j}\right\rangle\right)$ is multiplicative transitive if and only if

$$
\forall i, j, k:\left\{\begin{array}{l}
\mu_{i j} \cdot \mu_{j k} \cdot \mu_{k i}=\mu_{i k} \cdot \mu_{k j} \cdot \mu_{j i} \\
\left(1-\nu_{i j}\right) \cdot\left(1-\nu_{j k}\right) \cdot\left(1-\nu_{k i}\right)=\left(1-\nu_{i k}\right) \cdot\left(1-\nu_{k j}\right) \cdot\left(1-\nu_{j i}\right)
\end{array}\right.
$$

\subsection{Construction of Multiplicative Transitivity Based IRPRs}

Expression (7) can be used to estimate the intuitionistic preference value between a pair of alternatives $\left(x_{i}, x_{j}\right)$ with $(i<j)$ using another different intermediate alternative $x_{k}(k \neq i, j)$ as follows:

$$
m r_{i j}^{k-}=\frac{\mu_{i k} \cdot \mu_{k j} \cdot \mu_{j i}}{\mu_{j k} \cdot \mu_{k i}} ; \quad m r_{i j}^{k+}=1-\frac{\left(1-\nu_{i k}\right) \cdot\left(1-\nu_{k j}\right) \cdot\left(1-\nu_{j i}\right)}{\left(1-\nu_{j k}\right) \cdot\left(1-\nu_{k i}\right)},
$$

as long as the denominators are not zero.

We call $m r_{i j}^{k}=\left\langle m r_{i j}^{k-}, m r_{i j}^{k+}\right\rangle$ the partially multiplicative transitivity based estimated intuitionistic preference value of the pair of alternatives $\left(x_{i}, x_{j}\right)$ obtained using the intermediate alternative $x_{k}$.

Notice that both Equations in (7) are always true when two of the three subindexes are equal. Let $k=i$, if $\mu_{j i} \neq 0$ then $m r_{i j}^{i-}=\mu_{i j}$, while if $\nu_{j i} \neq 1$ then $m r_{i j}^{i+}=\nu_{i j}$. Because $\mu_{j i}=\nu_{i j}$, then we have that: $\mu_{j i} \neq 0$ if and only if $\nu_{i j} \neq 0$, and $\nu_{j i} \neq 1$ if and only if $\mu_{i j} \neq 1$. Thus, if $k=i$ and $\left(r_{i j}, r_{j i}\right) \neq(\langle 1,0\rangle,\langle 0,1\rangle)$ we have $r_{i j}=m r_{i j}^{i}$. Similarly, if $k=j$ and $\left(r_{i j}, r_{j i}\right) \neq(\langle 0,1\rangle,\langle 1,0\rangle)$ we have $r_{i j}=m r_{i j}^{j}$. Summarising, although it is possible to obtain the multiplicative transitivity based estimated intuitionistic preference value of the pair of alternatives $\left(x_{i}, x_{j}\right)$ when $k \in\{i, j\}$ and $\left(r_{i j}, r_{j i}\right) \neq\{(\langle 1,0\rangle,\langle 0,1\rangle),(\langle 0,1\rangle,\langle 1,0\rangle)\}$, it is also true that there is no indirect estimation process as described above. Furthermore, as we will see later in the paper, when the intuitionistic preference value $r_{i j}$ is unknown its estimation will automatically require that $k \notin\{i, j\}$. Finally, when $i=j$ we have by definition that $r_{i i}=\langle 0.5,0.5\rangle$ and we would have $m r_{i i}^{k}=r_{i i}$ whenever $r_{i k} \notin(\langle 0,1\rangle,\langle 1,0\rangle)$. Thus, this case will not be relevant in the case of having incomplete information, and it is also not assumed from now on.

The average of all possible partially multiplicative transitivity based estimated values of the pair of alternatives $\left(x_{i}, x_{j}\right)$ can be interpreted as their global multiplicative transitivity based estimated value

$$
m r_{i j}^{-}=\frac{\sum_{k \in R_{i j}^{01}} m r_{i j}^{k-}}{\# R_{i j}^{01}} ; \quad m r_{i j}^{+}=\frac{\sum_{k \in R_{i j}^{01}} m r_{i j}^{k+}}{\# R_{i j}^{01}},
$$

where $R_{i j}^{01}=\left\{k \neq i, j \mid\left(r_{i k}, r_{k j}\right) \notin R^{01}\right\}, R^{01}=\{(\langle 1,0\rangle,\langle 0,1\rangle),(\langle 0,1\rangle,\langle 1,0\rangle)\}$, and $\# R_{i j}^{01}$ is the cardinality of $R_{i j}^{01}$. 
Therefore, given an IRPR, $R=\left(r_{i j}\right)=\left(\left\langle\mu_{i j}, \nu_{i j}\right\rangle\right)$, the following multiplicative transitivity based IRPR, $M R=\left(\left\langle m r_{i j}^{-}, m r_{i j}^{+}\right\rangle\right)_{n \times n}$, can be constructed:

$$
m r_{i j}^{-}=\left\{\begin{array}{ll}
\frac{\sum_{k \in R_{i j}^{01}} m r_{i j}^{k-}}{\# R_{i j}^{01}}, & i<j \\
0.5, & i=j ; \\
\frac{\sum_{k \in R_{j i}^{01}} m r_{j i}^{k+}}{\# R_{j i}^{01}}, & i>j
\end{array} \quad m r_{i j}^{+}= \begin{cases}\frac{\sum_{k \in R_{i j}^{01}} m r_{i j}^{k+}}{\# R_{i j}^{01}}, & i<j \\
0.5, & i=j \\
\frac{\sum_{k \in R_{j i}^{01}} m r_{j i}^{k-}}{\# R_{j i}^{01}}, & i>j\end{cases}\right.
$$

\subsection{Consistency Indexes}

If an IRPR $R=\left(r_{i j}\right)=\left(\left\langle\mu_{i j}, \nu_{i j}\right\rangle\right)$ is multiplicative transitive then $R=M R$. Indeed, if $R$ is multiplicative transitive the two Equations in (7) are true $\forall i, j, k$. In particular, we have

$$
\mu_{i j}=\frac{\mu_{i k} \cdot \mu_{k j} \cdot \mu_{j i}}{\mu_{j k} \cdot \mu_{k i}} ; \quad \nu_{i j}=1-\frac{\left(1-\nu_{i k}\right) \cdot\left(1-\nu_{k j}\right) \cdot\left(1-\nu_{j i}\right)}{\left(1-\nu_{j k}\right) \cdot\left(1-\nu_{k i}\right)}
$$

whenever $k \in R_{i j}^{01}$. Consequently, $m r_{i j}^{k-}=\mu_{i j}$ and $m r_{i j}^{k+}=\nu_{i j}$ for all $i, j$ and $k \in R_{i j}^{01}$, which proves that $r_{i j}=m r_{i j}$ for all $i, j$. An IRPR $R$ will be referred to as multiplicative consistent from now on when $R=M R$.

Definition 9 (Multiplicative Consistent IRPR). An IRPR $R=\left(r_{i j}\right)=\left(\left\langle\mu_{i j}, \nu_{i j}\right\rangle\right)$ is multiplicative consistent if and only if $R=M R$.

In the following, we will propose a process to measure the degree of consistency of an IRPR, $R$, that is based on its similarity with the corresponding multiplicative transitivity based IRPR, $M R$. The similarity between the values $r_{i j}$ and $m r_{i j}$ is proposed to be used in measuring the level of consistency of an IRPR at its three different levels: pair of alternatives, alternatives and relation. A similarity function between IFSs is defined based on the use of a metric $(d)$ describing the distance between IFSs.

Level 1. Consistency Index of pair of alternatives.

$$
C I_{i j}=1-d\left(r_{i j}, m r_{i j}\right) \quad \forall i, j .
$$

The higher the value of $C I_{i j}$ the more consistent is $r_{i j}$ with respect to the rest of the preference values involving alternatives $x_{i}$ (row i of the IRPR) and $x_{j}$ (column $\mathrm{j}$ of the IRPR).

Level 2. Consistency Index of alternatives.

$$
C I_{i}=\frac{\sum_{j=1 ; i \neq j}^{n} C I_{i j}}{n-1} .
$$


Level 3. Consistency Index of an IRPR.

$$
C I=\frac{\sum_{i=1}^{n} C I_{i}}{n} .
$$

Note that because $d$ is a distance function, we always have that $C I_{i j}=C I_{j i}$ and therefore, the above expression can be simplified to:

$$
C I=\frac{2 \cdot \sum_{i, j=1 ; i<j}^{n} C I_{i j}}{n(n-1)} .
$$

Proposition 1. An IRPR $R$ is multiplicative consistent if and only if $C I=1$.

Proof.

1. $R$ is multiplicative consistent $\Longrightarrow C I=1$. Definition 9 implies that $R=M R$, and therefore $d\left(r_{i j}, m r_{i j}\right)=0 \forall i, j$. Consequently, $C I=1$.

2. $C I=1 \Longrightarrow R$ is multiplicative consistent. If $C I=1$ then $\sum_{i, j=1, i \neq j}^{n} C I_{i j}=n \times(n-1)$. Because $C I_{i j} \in[0,1]$ then $C I_{i j}=1 \forall i \neq j$. Therefore we have that $C I=1$ if and only if $r_{i j}=m r_{i j} \forall i \neq j$. Finally, when $i=j$ we have $m r_{i i}^{k}=r_{i i}=\langle 0.5,0.5\rangle$ whenever $r_{i k} \notin(\langle 0,1\rangle,\langle 1,0\rangle)$, and therefore $r_{i i}=m r_{i i}=\langle 0.5,0.5\rangle$. Thus, we have that $r_{i j}=m r_{i j} \forall i, j$. Therefore, $R=M R$, i.e. $R$ is multiplicative consistent.

Example 1. Given the IRPR

$$
\mathbf{R}=\left(\begin{array}{cccc}
\langle 0.5,0.5\rangle & \langle 0.4,0.3\rangle & \langle 0.5,0.4\rangle & \langle 0.4,0.5\rangle \\
\langle 0.3,0.4\rangle & \langle 0.5,0.5\rangle & \langle 0.5,0.4\rangle & \langle 0.3,0.4\rangle \\
\langle 0.4,0.5\rangle & \langle 0.4,0.5\rangle & \langle 0.5,0.5\rangle & \langle 0.3,0.4\rangle \\
\langle 0.5,0.4\rangle & \langle 0.4,0.3\rangle & \langle 0.4,0.3\rangle & \langle 0.5,0.5\rangle
\end{array}\right)
$$

The following multiplicative transitivity based IRPR is obtained:

$$
\mathbf{M R}=\left(\begin{array}{cccc}
\langle 0.50,0.50\rangle & \langle 0.31,0.41\rangle & \langle 0.55,0.41\rangle & \langle 0.48,0.39\rangle \\
\langle 0.41,0.31\rangle & \langle 0.50,0.50\rangle & \langle 0.39,0.49\rangle & \langle 0.31,0.39\rangle \\
\langle 0.41,0.55\rangle & \langle 0.49,0.39\rangle & \langle 0.50,0.50\rangle & \langle 0.25,0.51\rangle \\
\langle 0.39,0.48\rangle & \langle 0.39,0.31\rangle & \langle 0.51,0.25\rangle & \langle 0.50,0.50\rangle
\end{array}\right)
$$

We provide the computation of entry $m r_{12}=\langle 0.31,0.41\rangle$. The multiplicative transitivity based estimation of the intuitionistic preference value between alternatives $x_{1}$ and $x_{2}$ is obtained using the 
intermediate alternatives $x_{3}$ and $x_{4}$. Using (8), we have (rounding to 2 decimal places):

$$
\begin{aligned}
& m r_{12}^{3-}=\frac{\mu_{13} \cdot \mu_{32} \cdot \mu_{21}}{\mu_{23} \cdot \mu_{31}}=\frac{0.5 \cdot 0.4 \cdot 0.3}{0.5 \cdot 0.4}=0.3 ; \\
& m r_{12}^{4-}=\frac{\mu_{14} \cdot \mu_{42} \cdot \mu_{21}}{\mu_{24} \cdot \mu_{41}}=\frac{0.4 \cdot 0.4 \cdot 0.3}{0.3 \cdot 0.5}=0.32 \\
& m r_{12}^{3+}=1-\frac{\left(1-\nu_{13}\right) \cdot\left(1-\nu_{32}\right) \cdot\left(1-\nu_{21}\right)}{\left(1-\nu_{23}\right) \cdot\left(1-\nu_{31}\right)}=1-\frac{(1-0.4) \cdot(1-0.5) \cdot(1-0.4)}{(1-0.4) \cdot(1-0.5)}=0.4 ; \\
& m r_{12}^{4+}=1-\frac{\left(1-\nu_{14}\right) \cdot\left(1-\nu_{42}\right) \cdot\left(1-\nu_{21}\right)}{\left(1-\nu_{24}\right) \cdot\left(1-\nu_{41}\right)}=1-\frac{(1-0.5) \cdot(1-0.3) \cdot(1-0.4)}{(1-0.4) \cdot(1-0.4)}=0.42 .
\end{aligned}
$$

Thus:

$$
m r_{12}^{-}=\frac{0.3+0.32}{2}=0.31 ; \quad m r_{12}^{+}=\frac{0.4+0.42}{2}=0.41 .
$$

Using the Hamming distance [48]:

$$
d\left(r_{i j}, m r_{i j}\right)=\left\|r_{i j}-m r_{i j}\right\|=\frac{1}{2}\left(\left|r_{i j}^{-}-m r_{i j}^{-}\right|+\left|r_{i j}^{+}-m r_{i j}^{+}\right|\right),
$$

the following consistency index of the IRPR $R$ is obtained: $C I=0.93$.

\section{Incomplete IRPRs: Estimation of Unknown Values}

In real decision making problems, there might be cases where an expert would not be able to efficiently express any kind of preference degree between two or more of the available options. Formally, the concept of an incomplete IRPR can be expressed via the concept of a partial function [1]:

Definition 10. A function $f: X \longrightarrow Y$ is partial when not every element in the set $X$ necessarily maps to an element in the set $Y$. When every element from the set $X$ maps to one element of the set $Y$ then we have a total function.

Definition 11 (Incomplete IRPR). An incomplete IRPR $R$ on a set of alternatives $X$ is characterized by partial membership and non-membership functions.

The definition of an IRPR given in Section 2 includes both definitions of complete and incomplete IRPRs. However, as there is no risk of confusion between a complete and an incomplete IRPR, in this paper we refer to the first type as simply IRPR. It is assumed that for incomplete IRPRs, given a pair of alternatives $\left(x_{i}, x_{j}\right)$ for which $r_{i j}$ is not known, both membership and non-memberships will be unknown. In general the letter $x$ will be used when a particular entry of an incomplete IRPR is unknown/missing.

Given an IRPR $R=\left(r_{i j}\right)=\left(\left\langle\mu_{i j}, \nu_{i j}\right\rangle\right)$, two RPRs, $B^{L}=\left(b_{i j}^{L}\right)_{n \times n}$ and $B^{R}=\left(b_{i j}^{R}\right)_{n \times n}$, can be constructed

$$
\forall i, j=1,2, \ldots n: \quad b_{i j}^{L}=\left\{\begin{array}{ll}
\mu_{i j} & i<j \\
0.5 & i=j \\
1-\nu_{i j} & i>j
\end{array} ; \quad b_{i j}^{R}= \begin{cases}1-\nu_{i j} & i<j \\
0.5 & i=j \\
\mu_{i j} & i>j\end{cases}\right.
$$


Proof of reciprocity of $B^{L}$ is provided. Because $\mu_{j i}=\nu_{i j} \forall i, j \in\{1, \ldots, n\}$ we have:

- $i<j: b_{i j}^{L}+b_{j i}^{L}=\mu_{i j}+\left(1-\nu_{j i}\right)=\nu_{j i}+\left(1-\nu_{j i}\right)=1$.

- $i>j \Leftrightarrow j<i \Rightarrow b_{j i}^{L}+b_{i j}^{L}=1$.

Reciprocity of $B^{R}$ is equally proved. Both $B^{L}$ and $B^{R}$ are known as the decomposed RPRs of the IRPR $R$.

Recall that Tanino's multiplicative transitivity property is equivalent to the cardinal consistency property [12] with the andlike representable Cross Ratio uninorm [47]

$$
U(x, y)= \begin{cases}0, & (x, y) \in\{(0,1),(1,0)\} \\ \frac{x \cdot y}{x \cdot y+(1-x) \cdot(1-y)}, & \text { Otherwise }\end{cases}
$$

If $R$ is an incomplete IRPR, then a missing preference value $r_{i j}(i \neq j)$ can be partially estimated, using an intermediate alternative $x_{k}$, with the value $\left\langle\left(b_{i j}^{L}\right)^{k}, 1-\left(b_{i j}^{R}\right)^{k}\right\rangle$ :

$$
\left(b_{i j}^{L}\right)^{k}=U\left(b_{i k}^{L}, b_{k j}^{L}\right) ; \quad\left(b_{i j}^{R}\right)^{k}=U\left(b_{i k}^{R}, b_{k j}^{R}\right) .
$$

The following notation is introduced:

$$
\begin{aligned}
A & =\{(i, j) \mid i, j \in\{1, \ldots, n\} \cap i \neq j\} ; \\
M V & =\left\{(i, j) \mid r_{i j} \text { is unknown, }(i, j) \in A\right\} ; \\
E V & =A \backslash M V .
\end{aligned}
$$

$M V$ is the set of pairs of different alternatives for which the intuitionistic preference degree is unknown or missing; $E V$ is the set of pairs of different alternatives with known intuitionistic preference values. The global multiplicative transitivity based estimated value, $\left\langle b_{i j}^{L}, 1-b_{i j}^{R}\right\rangle$, is defined as follows:

$$
u b_{i j}^{L}=\frac{\sum_{k \in H_{i j}^{01}}\left(b_{i j}^{L}\right)^{k}}{\# H_{i j}^{01}} ; \quad u b_{i j}^{R}=\frac{\sum_{k \in H_{i j}^{01}}\left(b_{i j}^{R}\right)^{k}}{\# H_{i j}^{01}},
$$

where $H_{i j}^{01}=\left\{k \in R_{i j}^{01} \mid(i, j) \in M V \&(i, k),(k, j) \in E V\right\}$.

The iterative procedure to complete RPRs developed in [28] can be adapted to IRPRs using the multiplicative transitivity developed here, and the following example illustrates its application.

Example 2. Let $X=\left\{x_{1}, x_{2}, x_{3}, x_{4}\right\}$ be a set of alternatives evaluated by a decision maker against a particular criterion using an incomplete $\operatorname{IRPR}, R=\left(r_{i j}\right)$ :

$$
R=\left(\begin{array}{cccc}
\langle 0.50,0.50\rangle & \langle 0.40,0.30\rangle & x & x \\
\langle 0.30,0.40\rangle & \langle 0.50,0.50\rangle & \langle 0.50,0.40\rangle & x \\
x & \langle 0.40,0.50\rangle & \langle 0.50,0.50\rangle & \langle 0.30,0.40\rangle \\
x & x & \langle 0.40,0.30\rangle & \langle 0.50,0.50\rangle
\end{array}\right)
$$


The associated decomposed RPRs are:

$$
B^{L}=\left(\begin{array}{cccc}
0.50 & 0.40 & x & x \\
0.60 & 0.50 & 0.50 & x \\
x & 0.50 & 0.50 & 0.30 \\
x & x & 0.70 & 0.50
\end{array}\right) ; \quad B^{R}=\left(\begin{array}{cccc}
0.50 & 0.70 & x & x \\
0.30 & 0.50 & 0.60 & x \\
x & 0.40 & 0.50 & 0.60 \\
x & x & 0.40 & 0.50
\end{array}\right)
$$

Step 1: The set of elements that can be estimated at this stage are:

$$
E M V_{1}=\{(1,3),(2,4),(3,1),(4,2)\} .
$$

Notice that $(1,4)$ cannot be estimated at this step. Indeed, the estimation of element $(1,4)$ requires that at least one of the following pairs of preference values are known: $\{(1,2),(2,4)\},\{(1,3),(3,4)\}$. However, the preference values for $(2,4)$ and $(1,3)$ are unknown. The same applies to $(4,1)$, which cannot be estimated at this step because the preference values for $(4,2)$ and $(3,1)$ are unknown.

The computation of the estimated values $b_{13}^{L}$ and $b_{13}^{R}$ is given below. These values are estimated by using intermediate and different alternatives $k$ so that the chain of preference values $(1, k)$ and $(k, 3)$ are known. The only intermediate alternative to use at this step is $k=2$, for which we have (rounding to 2 decimal places):

$$
b_{13}^{L}=b_{13}^{L 2}=\frac{b_{12}^{L} \cdot b_{23}^{L}}{b_{12}^{L} \cdot b_{23}^{L}+\left(1-b_{12}^{L}\right) \cdot\left(1-b_{23}^{L}\right)}=\frac{0.4 \cdot 0.5}{0.4 \cdot 0.5+0.6 \cdot 0.5}=0.4
$$

and

$$
b_{13}^{R}=b_{13}^{R 2}=\frac{b_{12}^{R} \cdot b_{23}^{R}}{b_{12}^{R} \cdot b_{23}^{R}+\left(1-b_{12}^{R}\right) \cdot\left(1-b_{23}^{R}\right)}=\frac{0.7 \cdot 0.6}{0.7 \cdot 0.6+0.3 \cdot 0.4}=0.78 .
$$

After the estimation process is applied, we have:

$$
B^{L}=\left(\begin{array}{cccc}
0.50 & 0.40 & 0.40 & x \\
0.60 & 0.50 & 0.50 & 0.30 \\
0.60 & 0.50 & 0.50 & 0.30 \\
x & 0.70 & 0.70 & 0.50
\end{array}\right) ; \quad B^{R}=\left(\begin{array}{cccc}
0.50 & 0.70 & 0.78 & x \\
0.30 & 0.50 & 0.60 & 0.69 \\
0.22 & 0.40 & 0.50 & 0.60 \\
x & 0.31 & 0.40 & 0.50
\end{array}\right)
$$

Step 2: The remaining unknown elements can be estimated at this stage, $E M V_{2}=\{(1,4),(4,1)\}$. We elaborate the computation process of the estimated value for $b_{14}^{L}$ (rounding to 2 decimal places):

$$
\begin{gathered}
b_{14}^{L 2}=\frac{b_{12}^{L} \cdot b_{24}^{L}}{b_{12}^{L} \cdot b_{24}^{L}+\left(1-b_{12}^{L}\right) \cdot\left(1-b_{24}^{L}\right)}=\frac{0.4 \cdot 0.3}{0.4 \cdot 0.3+0.6 \cdot 0.7}=0.22 \\
b_{14}^{L 3}=\frac{b_{13}^{L} \cdot b_{34}^{L}}{b_{13}^{L} \cdot b_{34}^{L}-\left(1-b_{13}^{L}\right) \cdot\left(1-b_{34}^{L}\right)}=\frac{0.4 \cdot 0.3}{0.4 \cdot 0.3+0.6 \cdot 0.7}=0.22 \\
b_{14}^{L}=\frac{b_{14}^{L 2}+b_{14}^{L 3}}{2}=0.22 .
\end{gathered}
$$


The rest of values $b_{14}^{R}, b_{41}^{L}$ and $b_{41}^{R}$ can be estimated following a similar computation process to that of $b_{14}^{L}$. Thus, we obtain the following completed RPRs:

$$
U B^{L}=\left(\begin{array}{cccc}
0.50 & 0.40 & 0.40 & 0.22 \\
0.60 & 0.50 & 0.50 & 0.30 \\
0.60 & 0.50 & 0.50 & 0.30 \\
0.78 & 0.70 & 0.70 & 0.50
\end{array}\right) ; \quad U B^{R}=\left(\begin{array}{cccc}
0.50 & 0.70 & 0.78 & 0.81 \\
0.30 & 0.50 & 0.60 & 0.69 \\
0.22 & 0.40 & 0.50 & 0.60 \\
0.19 & 0.31 & 0.40 & 0.50
\end{array}\right)
$$

The complete multiplicative transitivity based IRPR is:

$$
U R=\left(\begin{array}{cccc}
\langle 0.50,0.50\rangle & \langle 0.40,0.30\rangle & \langle 0.40,0.22\rangle & \langle 0.22,0.19\rangle \\
\langle 0.30,0.40\rangle & \langle 0.50,0.50\rangle & \langle 0.50,0.40\rangle & \langle 0.30,0.31\rangle \\
\langle 0.22,0.40\rangle & \langle 0.40,0.50\rangle & \langle 0.50,0.50\rangle & \langle 0.30,0.40\rangle \\
\langle 0.19,0.22\rangle & \langle 0.31,0.30\rangle & \langle 0.40,0.30\rangle & \langle 0.50,0.50\rangle
\end{array}\right)
$$

It is important to establish conditions that guarantee that all the missing values of an incomplete preference relation can be estimated. Herrera-Viedma et al. in [28] provided sufficient conditions that guarantee the success of the above iterative estimation procedure. The general sufficient condition for an incomplete preference relation to be completed with this iterative estimation procedure is that a set of $(n-1)$ non-leading diagonal preference values, where each one of the alternatives is compared at least once, is known.

\section{Consensus Model with Incomplete IRPRs}

In the resolution process of a GDM, it is expected to associate a higher importance degree with the experts providing the most consistent information, with the aim of achieving a collective solution with maximum group consensus and a high level of consistency. To achieve these 'rational' criteria, a new multiplicative consistency induced ordered weighted averaging (MC-IOWA) operator to aggregate the individual IRPRs is proposed and introduced. The MC-IOWA operator associated weighting vector is derived using the individual experts' multiplicative consistency index (CI) as defined in Subsection 2.4, providing a monotonic increasing mapping between the experts' consistency levels and their contribution weight in the collective IRPR, and ultimately in the solution of the GDM problem. Once the collective IRPR is obtained, a proximity index (PI) measuring the level of agreement between the individual and collective preferences is computed. The consensus degree is defined taking into account both CI and PI. When the consensus level reaches a threshold value, agreed by the group of experts, the resolution process of the GDM is carried out; otherwise a feedback mechanism is activated, and personalised recommendations generated to support the individual experts, until the threshold level of consensus is achieved. The feedback recommendations will help the experts to identify the preference 


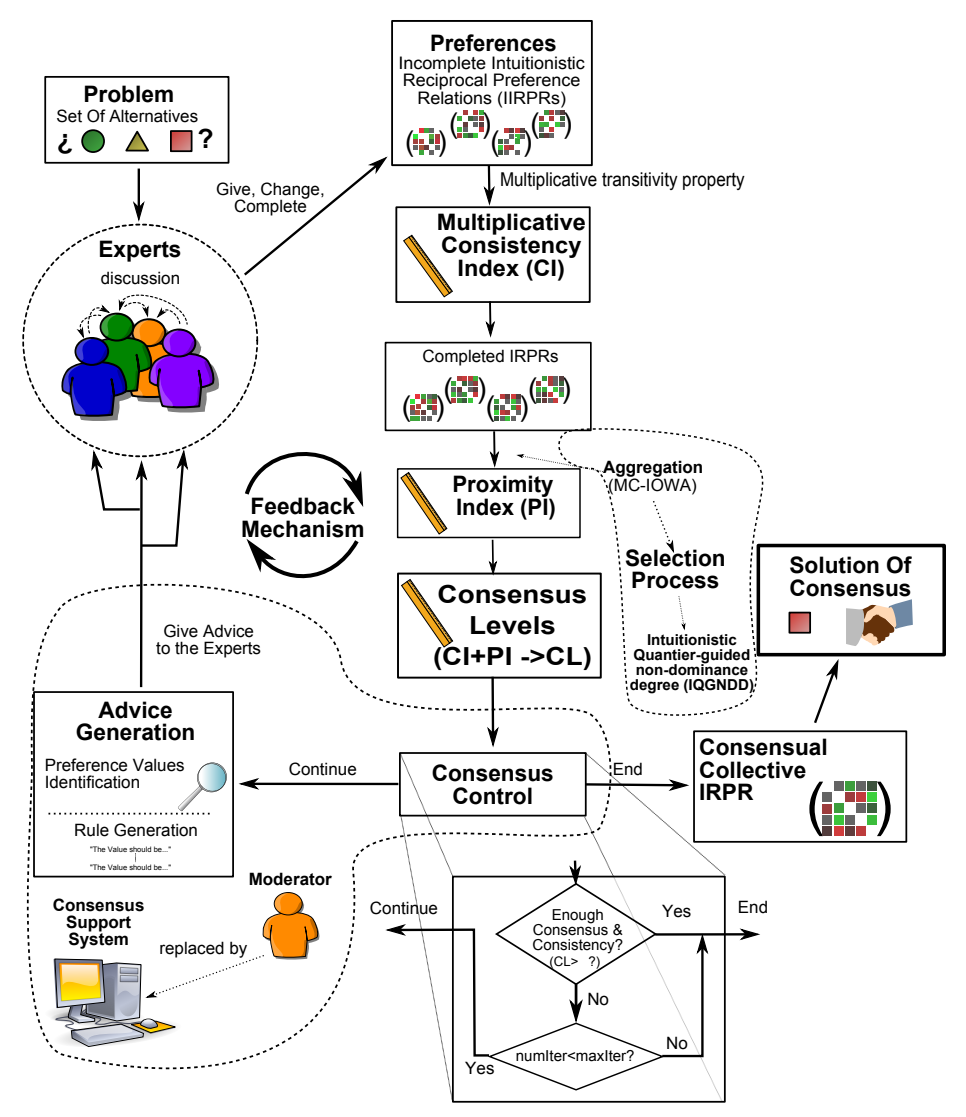

Figure 2: Consensus Model with Incomplete IRPRs

values that should be considered for changing. The recommendations will also include the values the experts should use to increase the level of agreement in a consistent way.

The consensus model with incomplete IRPRs is illustrated in Figure 2. It consists of the following six steps: (1) Estimating missing values; (2) Computing consistency indexes; (3) Computing proximity indexes; (4) Computing consensus levels; (5) Feedback mechanism; and (6) Selection process. The first two steps have already been covered in Sections 2 and 3, respectively. The remaining steps will be presented in detail in following subsections.

\subsection{Computing Proximity Indexes}

The proximity degrees will measure how close the individual preferences are from the group or collective preferences. The collective preferences are obtained by fusing all the individuals' preferences using the multiplicative consistency induced ordered weighted averaging (MC-IOWA) operator [11, 38], which extends the induced ordered weighted averaging (IOWA) operator proposed by Yager [46]:

Definition 12. An IOWA operator of dimension $m$ is a function $\Phi_{W}:(\mathbb{R} \times \mathbb{R})^{m} \longrightarrow \mathbb{R}$, to which a set of weights or weighting vector is associated, $W=\left(w_{1}, \ldots, w_{m}\right)$, such that $w_{i} \in[0,1]$ and $\Sigma_{i} w_{i}=1$, is expressed as follows:

$$
\Phi_{W}\left(\left\langle u_{1}, p_{1}\right\rangle, \ldots,\left\langle u_{m}, p_{m}\right\rangle\right)=\sum_{i=1}^{m} w_{i} \cdot p_{\sigma(i)}
$$


being $\sigma:\{1, \ldots, m\} \longrightarrow\{1, \ldots, m\}$ a permutation such that $u_{\sigma(i)} \geq u_{\sigma(i+1)}, \forall i=1, \ldots, m-1$.

In our decision-making context, each expert can always be associated his/her IRPR consistency index value. The more consistent the preferences provided by an expert are, the more importance should be placed on that expert. In other words, we propose to use the consistency indexes to establish the ordering of the preference values to be aggregated, in which case we would be implementing the concept of consistency in the aggregation process of our decision-making model [11]:

Definition 13 (MC-IOWA operator). Let a set of experts, $E=\left\{e_{1}, \ldots, e_{m}\right\}$, provide preferences about a set of alternatives, $X=\left\{x_{1}, \ldots, x_{n}\right\}$, using the IRPRs, $\left\{R^{1}, \ldots, R^{m}\right\}$. A MC-IOWA operator of dimension $m, \Phi_{W}^{C}$, is an IOWA operator whose set of order inducing values is the set of consistency index values, $\left\{C I^{1}, \ldots, C I^{m}\right\}$, associated with the set of experts.

Therefore, the collective IRPR $R^{c}=\left(r_{i j}^{c}\right)=\left(\left\langle\mu_{i j}^{c}, \nu_{i j}^{c}\right\rangle\right)$ is computed as follows:

$$
\begin{aligned}
& \mu_{i j}^{c}=\Phi_{W}^{C}\left(\left\langle C I^{1}, \mu_{i j}^{1}\right\rangle, \cdots,\left\langle C I^{m}, \mu_{i j}^{m}\right\rangle\right)=\sum_{h=1}^{m} \gamma_{\sigma(h)} \cdot \mu_{i j}^{\sigma(h)} \\
& \nu_{i j}^{c}=\Phi_{W}^{C}\left(\left\langle C I^{1}, \nu_{i j}^{1}\right\rangle, \cdots,\left\langle C I^{m}, \nu_{i j}^{m}\right\rangle\right)=\sum_{h=1}^{m} \gamma_{\sigma(h)} \cdot \nu_{i j}^{\sigma(h)}
\end{aligned}
$$

with $C I^{\sigma(h-1)} \geq C I^{\sigma(h)}, \gamma_{\sigma(h-1)} \geq \gamma_{\sigma(h)} \geq 0(\forall h \in\{2, \cdots, m\})$ and $\sum_{h=1}^{m} \gamma_{\sigma(h)}=1$.

The general procedure for the inclusion of importance weight values in the aggregation process involves the transformation of the values to aggregate, $r_{i j}^{h}$, under the importance degree $u_{h}$ to generate a new value, $\bar{r}_{i j}^{h}$, and then aggregate these new values using an aggregation operator. In the area of quantifier guided aggregations, Yager provided a procedure to evaluate the overall satisfaction of $m$ important criteria (experts) by an alternative $x$ by computing the weighting vector associated to an OWA operator as follows [45]:

$$
w_{h}=Q\left(\frac{S(h)}{S(m)}\right)-Q\left(\frac{S(h-1)}{S(m)}\right)
$$

being $Q$ the membership function of the linguistic quantifier, $S(h)=\sum_{k=1}^{h} u_{\sigma(k)}$, and $\sigma$ the permutation used to produce the ordering of the values to be aggregated. This approach for the inclusion of importance degrees associates a zero weight to those experts with zero importance degree. The linguistic quantifier is a Basic Unit-interval Monotone (BUM) function $Q:[0,1] \rightarrow[0,1]$ such that $Q(0)=0, Q(1)=1$ and if $x>y$ then $Q(x) \geq Q(y)$.

Yager extended this procedure to the case of IOWA operator. In this case, each component in the aggregation consists of a triple $\left(r_{i j}^{h}, u_{h}, v_{h}\right)$ where $r_{i j}^{h}$ is the argument value to aggregate, $u_{h}$ is the importance weight value associated to $r_{i j}^{h}$, and $v_{h}$ is the order inducing value [46]. The same expression as above is used, and $\sigma$ is the permutation such that $v_{\sigma(h)}$ is the $h$-th largest value in the set $\left\{v_{1}, \ldots, v_{m}\right\}$. 
In our case, we propose to use the consistency values associated with each of the expert both as an importance weight associated to the argument and as the order inducing values $u_{h}=v_{h}=C I^{h}$. Thus, the ordering of the preference values is first induced by the ordering of the experts from the most to the least consistent, and the weights of the MC-IOWA operator is obtained by applying the above expression (12), which reduces to

$$
\gamma_{\sigma(h)}=Q\left(\frac{S(\sigma(h))}{S(\sigma(m))}\right)-Q\left(\frac{S(\sigma(h-1))}{S(\sigma(m))}\right)
$$

with $\left.S(\sigma(h))=\sum_{k=1}^{h} C I^{\sigma(k)}\right)$, and $C I^{\sigma(h)}$ is the $h$-th largest value of set $\left\{C I^{1}, \ldots, C I^{m}\right\}$.

The BUM function guarantees that all individuals contribute to the final aggregated value because it is a strictly increasing function. To guarantee that the higher the consistency index, the higher the weighting value associated with it, i.e. for the following to be verified

$$
C I^{\sigma(1)} \geq C I^{\sigma(2)} \geq \ldots \geq C I^{\sigma(m)} \geq 0 \Rightarrow \gamma_{\sigma(1)} \geq \gamma_{\sigma(2)} \ldots \geq \gamma_{\sigma(m)} \geq 0
$$

additional constraints are to be imposed to the BUM function. In [11], it was proven that it is sufficient for the BUM function to be concave for the above to be true.

The metric used to compute consistency indexes is used here to compute the proximity (similarity) between an individual IRPR, $R^{h}=\left(r_{i j}^{h}\right)$, and the collective IRPR, $R^{c}=\left(r_{i j}^{c}\right)$, at the three different levels of the relation:

Level 1. Proximity index on pairs of alternatives. The proximity of an expert, $e_{h}$, preference value on the pair of alternatives $\left(x_{i}, x_{k}\right)$ to the group one, denoted $P P_{i k}^{h}$, is defined as:

$$
P P_{i j}^{h}=1-d\left(r_{i j}^{h}, r_{i j}^{c}\right)
$$

Level 2. Proximity index on alternatives. The proximity of an expert, $e_{h}$, preferences involving the alternative $x_{i}$ to the group ones, denoted $P A_{i}^{h}$, is defined as:

$$
P A_{i}^{h}=\frac{\sum_{j=1 ; j \neq i}^{n}\left(P P_{i j}^{h}+P P_{j i}^{h}\right)}{2(n-1)}
$$

Level 3. Proximity index on the relation. The proximity of an expert, $e_{h}$, preference relation to the group one, denoted $P I^{h}$, is defined as:

$$
P I^{h}=\frac{\sum_{i=1}^{n} P A_{i}^{h}}{n}
$$

\subsection{Computing Consensus Levels}

Given an IRPR, $R$, its consensus level (CL) is defined as follows:

$$
C L=\delta \cdot C I+(1-\delta) \cdot P I
$$


where $\delta \in[0,1]$ is a parameter to control the weight of both consistency and proximity criteria. Similar expressions apply to $C L_{i}$ and $C L_{i j}$, respectively. A value of $\delta>0.5$ is used to provide more importance to the consistency index in the computation of the consensus degrees. The particular value to use will obviously depend on the group of experts and the importance they would like to give to the consistency criteria in the decision-making process.

The consensus is defined as the full and unanimous agreement of all the experts regarding all the feasible alternatives. However, it is inconvenient because it only allows differentiating between two states, namely, the existence and absence of consensus. Also, the chances for reaching such a full agreement are rather low. Therefore, the following restriction can be imposed: $\gamma<1$. Additionally, in most cases when more than half of people agree the decision-making output may be acceptable. Thus, the following restriction could also be imposed: $\gamma \geq 0.5$. Consequently, we can assume that the threshold value $\gamma \in[0.5,1)$.

The consensus levels can be used to decide when the feedback mechanism should be applied to give advice to the experts, or when the consensus reaching process has to come to an end. When $C L^{h}(h=1, \ldots, m)$ satisfies a minimum satisfaction threshold value $\gamma \in[0.5,1)$, then the consensus reaching process ends, and the selection process is applied to achieve the solution of consensus.

\subsection{Feedback Mechanism}

When at least one of the experts' consensus levels is below the fixed threshold value, a feedback mechanism is activated to generate personalised advice to those experts. This activity includes two steps: Identification of the preference values that should be changed and Generation of advice.

\subsubsection{Identification of the Preference Values}

The preference values that are contributing less to the consensus are identified. To do that, the following three step identification procedure that uses the proximity and consistency indexes is carried out:

Step 1. The experts with a consensus level lower than the threshold value $\gamma$ are identified:

$$
E X P C H=\left\{h \mid C L^{h}<\gamma\right\}
$$

Step 2. For the identified experts, their alternatives with a consensus level lower than the satisfaction threshold $\gamma$ are identified:

$$
A L T=\left\{(h, i) \mid e_{h} \in E X P C H \& C L_{i}^{h}<\gamma\right\} .
$$

Step 3. Finally, the preference values to be changed are:

$$
A P S=\left\{(h, i, k) \mid(h, i) \in A L T \& C L_{i k}^{h}<\gamma\right\}
$$




\subsubsection{Generation of Advice}

The feedback mechanism generates personalised recommendation rules, which will tell the experts the preference values they should change and the new preference values to use in order to increase their consensus level. For all $(h, i, j) \in A P S$, the personalised recommendation rules are identified as follow:

1. If $(i, j) \in E V^{h}$ the recommendation generated for expert $e_{h}$ is: "You should change your preference value for the pair of alternatives $(i, j), r_{i j}^{h}=\left\langle\mu_{i j}^{h}, \nu_{i j}^{h}\right\rangle$, to a value closer to $r r_{i j}^{h}=$ $\left\langle r \mu_{i j}^{h}, r \nu_{i j}^{h}\right\rangle . "$

2. If $(i, j) \in M V^{h}$ the recommendation generated for expert $e_{h}$ is: "Your missing preference value for the pair of alternatives $(i, j)$ should be as close as possible to $r r_{i j}^{h}=\left\langle r \mu_{i j}^{h}, r \nu_{i j}^{h}\right\rangle$."

$$
\left\langle r \mu_{i j}^{h}, r \nu_{i j}^{h}\right\rangle=\left\langle\delta \cdot \mu_{i j}^{h}+(1-\delta) \cdot \mu_{i j}^{c}, \delta \cdot \nu_{i j}^{h}+(1-\delta) \cdot \nu_{i j}^{c}\right\rangle
$$

It is worth noting that if the recommended values are implemented then the new preference values will be closer to the collective ones. Therefore, the production of recommendations to all experts, and their implementation, will guarantee that all expert consensus index values increase, subject to the following additional condition: the consistency index values to use in all of the consensus rounds are the same. This is proved in the following:

Lemma 1. Let $\left\{R^{h}=\left(r_{i j}^{h}\right)=\left(\left\langle\mu_{i j}^{h}, \nu_{i j}^{h}\right\rangle\right) \mid h=1, \cdots, m\right\}$ be a set of IRPRs and $R^{c}=\left(r_{i j}^{c}\right)=$ $\left(\left\langle\mu_{i j}^{c}, \nu_{i j}^{c}\right\rangle\right)$ be the collective IRPR obtained using the MC-IOWA operator whose order is induced using the associated set of consistency index values $\left\{C L^{h} \mid h=1, \cdots, m\right\}$. Let $\left\{r R^{h}=\left(r r_{i j}^{h}\right) \mid h=1, \cdots, m\right\}$ be the set of new IRPRs with

$$
\left\langle r \mu_{i j}^{h}, r \nu_{i j}^{h}\right\rangle=\left\langle\delta \cdot \mu_{i j}^{h}+(1-\delta) \cdot \mu_{i j}^{c}, \quad \delta \cdot \nu_{i j}^{h}+(1-\delta) \cdot \nu_{i j}^{c}\right\rangle
$$

and $r R^{c}=\left(r r_{i j}^{c}\right)=\left(\left\langle r \mu_{i j}^{c}, r \nu_{i j}^{c}\right\rangle\right)$ the collective IRPR obtained using the same MC-IOWA used for $R^{c}$. We have: $R^{c}=r R^{c}$.

Proof. Notice that the MC-IOWA operator is a weighted average operator with the same weights associated to all the elements of the same IRPR, and therefore we have:

$$
\mu_{i j}^{c}=\sum_{h=1}^{m} \gamma_{\sigma(h)} \cdot \mu_{i j}^{\sigma(h)} ; \quad \nu_{i j}^{c}=\sum_{h=1}^{m} \gamma_{\sigma(h)} \cdot \nu_{i j}^{\sigma(h)}
$$

with $\left.\gamma_{\sigma(h)}=Q\left(\frac{S(\sigma(h))}{S(\sigma(m))}\right)-Q\left(\frac{S(\sigma(h-1))}{S(\sigma(m))}\right) ; \quad S(\sigma(h))=\sum_{k=1}^{h} C I^{\sigma(k)}\right), Q$ is a BUM function and $C I^{\sigma(h)}$ is the $h$-th largest value of set $\left\{C I^{1}, \ldots, C I^{m}\right\}$. We have that $r \mu_{i j}=\delta \cdot \mu_{i j}+(1-\delta) \cdot \mu_{i j}^{c}$ and $r \nu_{i j}=\delta \cdot \nu_{i j}+(1-\delta) \cdot \nu_{i j}^{c}$. Therefore:

$$
\begin{aligned}
r \mu_{i j}^{c} & =\sum_{h=1}^{m} \gamma_{\sigma(h)} \cdot r \mu_{i j}^{\sigma(h)}=\sum_{h=1}^{m} \gamma_{\sigma(h)} \cdot\left[\delta \cdot \mu_{i j}^{\sigma(h)}+(1-\delta) \cdot \mu_{i j}^{c}\right] \\
& =\delta \cdot \sum_{h=1}^{m} \gamma_{\sigma(h)} \cdot \mu_{i j}^{\sigma(h)}+(1-\delta) \cdot \mu_{i j}^{c}=\delta \cdot \mu_{i j}^{c}+(1-\delta) \cdot \mu_{i j}^{c}=\mu_{i j}^{c}
\end{aligned}
$$


Similarly, we can prove that $r \nu_{i j}^{c}=\nu_{i j}^{c}$, which means that $R^{c}=r R^{c}$.

Proposition 2. Under the condition of Lemma 1 we have: $d\left(r r_{i j}^{h}, r r_{i j}^{c}\right) \leq d\left(r_{i j}^{h}, r_{i j}^{c}\right)$.

Proof. Because $d\left(r_{i j}^{h}, r_{i j}^{c}\right)=\frac{1}{2}\left(\left|\mu_{i j}^{h}-\mu_{i j}^{c}\right|+\left|\nu_{i j}^{h}-\nu_{i j}^{c}\right|\right)$, to prove that $d\left(r r_{i j}^{h}, r r_{i j}^{c}\right) \leq d\left(r_{i j}^{h}, r_{i j}^{c}\right)$ we only need to prove that $d\left(r r_{i j}^{h-}, r r_{i j}^{c-}\right) \leq d\left(r_{i j}^{h-}, r_{i j}^{c-}\right)$ and $d\left(r r_{i j}^{h+}, r r_{i j}^{c+}\right) \leq d\left(r_{i j}^{h+}, r_{i j}^{c+}\right)$ with $d\left(r r_{i j}^{h-}, r r_{i j}^{c-}\right)=$ $\left\|r \mu_{i j}^{h}-r \mu_{i j}^{c}\right\|$ and $d\left(r r_{i j}^{h+}, r r_{i j}^{c+}\right)=\left\|r \nu_{i j}^{h}-r \nu_{i j}^{c}\right\|$. Applying Lemma 1, we have:

$$
d\left(r r_{i j}^{h-}, r r_{i j}^{c-}\right)=\left\|\delta \cdot \mu_{i j}^{h}+(1-\delta) \cdot \mu_{i j}^{c}-\mu_{i j}^{c}\right\|=\delta \cdot\left\|\mu_{i j}^{h}-\mu_{i j}^{c}\right\| \leq d\left(r_{i j}^{h-}, r_{i j}^{c-}\right) .
$$

Similarly, we can prove that $d\left(r r_{i j}^{h+}, r r_{i j}^{c+}\right) \leq d\left(r_{i j}^{h+}, r_{i j}^{c+}\right)$.

Because $d\left(r r_{i j}^{h-}, r r_{i j}^{c-}\right)=\delta \cdot d\left(r_{i j}^{h-}, r_{i j}^{c-}\right)$, we have that the feedback mechanism would make the consensus process to converge to a unanimous consensus for all $\delta<1$. The above results are valid when all experts change all their preference values to the feedback recommendation values. Therefore, the production of recommendations to all experts, and their implementation, will guarantee that all expert consensus index values increase, subject to the following additional condition: the consistency index values to use in all of the consensus rounds are the same. This is graphically illustrated in Figure 3(a). However, in real applications all experts will not receive feedback recommendations, otherwise the computational complexity of the decision making process would increase. Therefore, in the consensus process proposed here only those experts with a consensus level lower than the threshold value will receive recommendations. In the final illustrative example, new consistency index values were computed once expert $e_{3}$ changed his/her preference values, which means that the previous results are not applicable. Indeed, the collective preference relation is not the same as that in the previous consensus round, which reflects that not all the experts changed their preference values. Thus, in this case the experts that change their preference values will become closer to the new collective preference relation than before, and in turn it would lead to an increase of their consensus level. Obviously, the same conclusion cannot be drawn for the experts that do not change their preference values. The application and implementation of the feedback mechanism in the final illustrative example is graphically illustrated in Figure 3(b).

\subsection{Selection Process}

Given an IRPR $R=\left(r_{i j}\right)=\left(\left\langle\mu_{i j}, \nu_{i j}\right\rangle\right)$, Wu and Chiclana developed a score function, $S_{W C}\left(r_{i j}\right)=$ $\left(\mu_{i j}-\nu_{i j}+1\right) / 2$, that can be interpreted as the membership function of a RPR known as the ScoreRPR (SRPR) associated to the IRPR [39]. Also, based on the OWA operator and the BUM function $Q$, Chiclana et al. presented the quantifier guided non-dominance degree for a RPR [10]. Therefore, it is possible to implement the concept of non-dominance to the case of an IRPR by using the associated SRPR. 


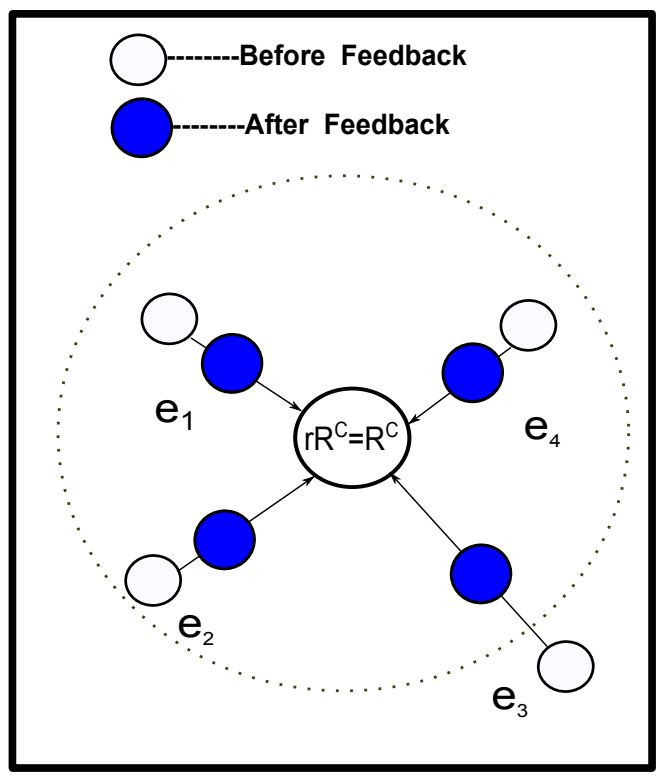

(a) All experts change preference values

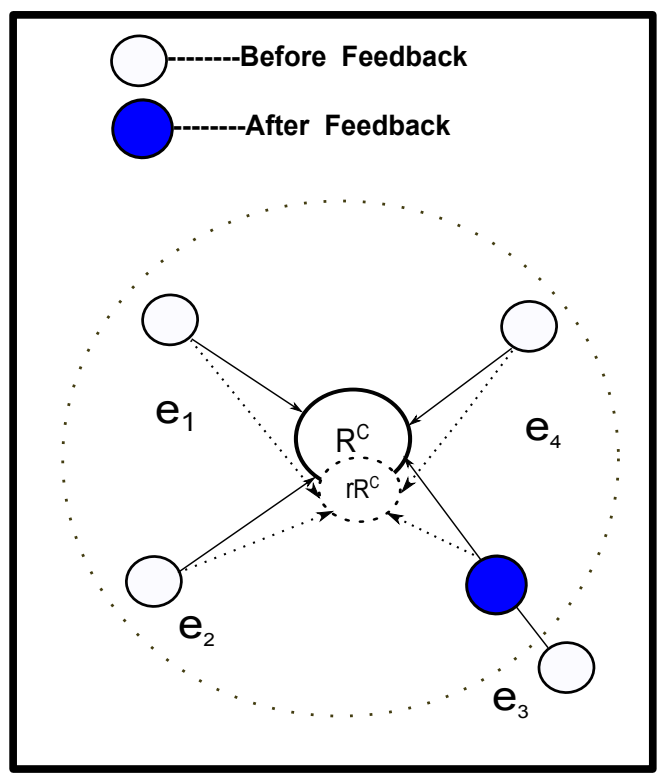

(b) Some experts change preference values

Figure 3: Consensus process before and after feedback mechanism. Left: All experts change preferences provided by feedback mechanism; Right: Only experts with consensus level below threshold value change preferences provided by feedback mechanism.

Definition 14 (Intuitionistic Quantifier Guided Non-Dominance Degree (IQGNDD)). Let $X=\left\{x_{1}, \ldots, x_{n}\right\}$ be a set of alternatives evaluated by a decision makeragainst a particular criterion using an IRPR $R=\left(r_{i j}\right)$, and $Q$ a BUM function. The intuitionistic quantifier guided non-dominance degree associated to the alternative $x_{i}, I Q G N D D_{i}$, is defined as follows:

$$
I Q G N D D_{i}=\psi_{Q}\left(1-p_{i j}^{s} ; j \neq i\right) \text {. }
$$

with $p_{i j}^{s}=\max \left\{p_{j i}-p_{i j}, 0\right\}$ representing the degree up to which to which $x_{i}$ is strictly dominated by $x_{j}, p_{i j}=S_{W C}\left(r_{i j}\right)$ and $\psi_{Q}$ is an OWA operator guided by the linguistic quantifier represented by the BUM function $Q$.

\section{Numerical example}

In selecting the most appropriate supplier for one of the key elements in its manufacturing process, a company employs the following four criteria: (1) Product quality; (2) Price; (3) Delivery performance; (4) Environment management.To determine the importance of these criteria, four different experts $\left\{e_{1}, e_{2}, e_{3}, e_{4}\right\}$ provide the following incomplete IRPRs over a set of four criteria $\left\{x_{1}, x_{2}, x_{3}, x_{4}\right\}$ :

$$
R^{\mathbf{1}}=\left(\begin{array}{cccc}
\langle 0.50,0.50\rangle & \langle 0.30,0.40\rangle & x & x \\
\langle 0.40,0.30\rangle & \langle 0.50,0.50\rangle & \langle 0.40,0.50\rangle & x \\
x & \langle 0.50,0.40\rangle & \langle 0.50,0.50\rangle & \langle 0.40,0.30\rangle \\
x & x & \langle 0.30,0.40\rangle & \langle 0.50,0.50\rangle
\end{array}\right)
$$




$$
\begin{aligned}
R^{2} & =\left(\begin{array}{cccc}
\langle 0.50,0.50\rangle & \langle 0.40,0.45\rangle & x & \langle 0.30,0.40\rangle \\
\langle 0.45,0.40\rangle & \langle 0.50,0.50\rangle & \langle 0.45,0.40\rangle & x \\
x & \langle 0.40,0.45\rangle & \langle 0.50,0.50\rangle & \langle 0.40,0.55\rangle \\
\langle 0.40,0.30\rangle & x & \langle 0.55,0.40\rangle & \langle 0.50,0.50\rangle
\end{array}\right) \\
R^{3} & =\left(\begin{array}{cccc}
\langle 0.50,0.50\rangle & \langle 0.50,0.40\rangle & x & \langle 0.40,0.30\rangle \\
\langle 0.40,0.50\rangle & \langle 0.50,0.50\rangle & \langle 0.60,0.30\rangle & \langle 0.50,0.40\rangle \\
x & \langle 0.30,0.60\rangle & \langle 0.50,0.50\rangle & \langle 0.35,0.40\rangle \\
\langle 0.30,0.40\rangle & \langle 0.40,0.50\rangle & \langle 0.40,0.35\rangle & \langle 0.50,0.50\rangle
\end{array}\right) \\
R^{4} & =\left(\begin{array}{cccc}
\langle 0.50,0.50\rangle & \langle 0.40,0.50\rangle & \langle 0.45,0.40\rangle & x \\
\langle 0.50,0.40\rangle & \langle 0.50,0.50\rangle & \langle 0.50,0.40\rangle & \langle 0.50,0.30\rangle \\
\langle 0.40,0.45\rangle & \langle 0.40,0.50\rangle & \langle 0.50,0.50\rangle & \langle 0.50,0.40\rangle \\
x & \langle 0.30,0.50\rangle & \langle 0.40,0.50\rangle & \langle 0.50,0.50\rangle
\end{array}\right)
\end{aligned}
$$

Step 1. Estimating Missing Values: The incomplete IRPRs are completed using the procedure presented in Section 3. Therefore, for each incomplete IRPR, $R^{h}$, a complete IRPR, $U R^{h}$, is obtained. The completed IRPR, $U R^{1}$, was already computed in Example 2. Similarly, the other completed IRPRs are derived, which results in the following:

$$
\begin{aligned}
U R^{1}= & \left(\begin{array}{llll}
\langle 0.50,0.50\rangle & \langle 0.30,0.40\rangle & \langle 0.22,0.40\rangle & \langle 0.19,0.22\rangle \\
\langle 0.40,0.30\rangle & \langle 0.50,0.50\rangle & \langle 0.40,0.50\rangle & \langle 0.31,0.30\rangle \\
\langle 0.40,0.22\rangle & \langle 0.50,0.40\rangle & \langle 0.50,0.50\rangle & \langle 0.40,0.30\rangle \\
\langle 0.22,0.19\rangle & \langle 0.30,0.31\rangle & \langle 0.30,0.40\rangle & \langle 0.50,0.50\rangle
\end{array}\right) \\
U R^{2}= & \left(\begin{array}{llll}
\langle 0.50,0.50\rangle & \langle 0.40,0.45\rangle & \langle 0.44,0.35\rangle & \langle 0.30,0.40\rangle \\
\langle 0.45,0.40\rangle & \langle 0.50,0.50\rangle & \langle 0.45,0.40\rangle & \langle 0.44,0.45\rangle \\
\langle 0.35,0.44\rangle & \langle 0.40,0.45\rangle & \langle 0.50,0.50\rangle & \langle 0.40,0.55\rangle \\
\langle 0.40,0.30\rangle & \langle 0.45,0.44\rangle & \langle 0.55,0.40\rangle & \langle 0.50,0.50\rangle
\end{array}\right) \\
U R^{3}= & \left(\begin{array}{lllll}
\langle 0.50,0.50\rangle & \langle 0.50,0.40\rangle & \langle 0.60,0.22\rangle & \langle 0.40,0.30\rangle \\
\langle 0.40,0.50\rangle & \langle 0.50,0.50\rangle & \langle 0.60,0.30\rangle & \langle 0.50,0.40\rangle \\
\langle 0.22,0.60\rangle & \langle 0.30,0.60\rangle & \langle 0.50,0.50\rangle & \langle 0.35,0.60\rangle \\
\langle 0.30,0.40\rangle & \langle 0.40,0.50\rangle & \langle 0.60,0.35\rangle & \langle 0.50,0.50\rangle
\end{array}\right) \\
U R^{4}= & \left(\begin{array}{llll}
\langle 0.50,0.50\rangle & \langle 0.40,0.50\rangle & \langle 0.45,0.40\rangle & \langle 0.42,0.31\rangle \\
\langle 0.50,0.40\rangle & \langle 0.50,0.50\rangle & \langle 0.50,0.40\rangle & \langle 0.50,0.30\rangle \\
\langle 0.40,0.45\rangle & \langle 0.40,0.50\rangle & \langle 0.50,0.50\rangle & \langle 0.50,0.40\rangle \\
\langle 0.31,0.42\rangle & \langle 0.30,0.50\rangle & \langle 0.40,0.50\rangle & \langle 0.50,0.50\rangle
\end{array}\right)
\end{aligned}
$$

Step 2. Computing Consistency Indexes: For each completed IRPR, $U R^{h}$, we obtain its associated multiplicative transitivity based IRPR, $M R^{h}$, as per the process given in Section 2.3, and 
compute the corresponding consistency indexes at the three different levels of a relation using the expressions given in Subsection 2.4: pair of alternatives, $C I_{i j}^{h}$; alternatives, $C I_{i}^{h}$; and relation, $C I^{h}$.

Level 1. The pair of alternatives level consistency indexes are:

$$
\begin{aligned}
& \left(C I_{i j}^{1}\right)=\left(\begin{array}{llll}
1.000 & 0.980 & 0.975 & 0.980 \\
0.980 & 1.000 & 0.965 & 0.965 \\
0.975 & 0.965 & 1.000 & 0.980 \\
0.980 & 0.965 & 0.980 & 1.000
\end{array}\right) \quad\left(C I_{i j}^{2}\right)=\left(\begin{array}{llll}
1.000 & 0.975 & 0.940 & 0.965 \\
0.975 & 1.000 & 0.915 & 0.960 \\
0.940 & 0.915 & 1.000 & 0.965 \\
0.965 & 0.960 & 0.965 & 1.000
\end{array}\right) \\
& \left(C I_{i j}^{3}\right)=\left(\begin{array}{llll}
1.000 & 0.945 & 0.970 & 0.925 \\
0.945 & 1.000 & 0.960 & 0.925 \\
0.970 & 0.960 & 1.000 & 0.990 \\
0.925 & 0.925 & 0.990 & 1.000
\end{array}\right)
\end{aligned}
$$

Level 2. The alternatives level consistency indexes are:

$$
\begin{array}{ll}
\left(C I_{i}^{1}\right)=(0.978,0.970,0.973,0.975) ; & \left(C I_{i}^{2}\right)=(0.960,0.950,0.940,0.963) \\
\left(C I_{i}^{3}\right)=(0.947,0.943,0.973,0.947) ; & \left(C I_{i}^{4}\right)=(0.958,0.950,0.985,0.977) .
\end{array}
$$

Level 3. The individual consistency indexes are: $C I^{1}=0.974, C I^{2}=0.953, C I^{3}=0.956, C I^{4}=$ 0.968 .

Using the consistency levels we have: $\sigma(1)=1, \sigma(2)=4, \sigma(3)=3$ and $\sigma(4)=2$. Using the concave BUM function $Q=r^{1 / 2}$, which can be used to represent the linguistic majority ' $m o s t$ of', we obtain the following weights $\lambda_{\sigma(1)}=0.50, \lambda_{\sigma(2)}=0.21, \lambda_{\sigma(3)}=0.16, \lambda_{\sigma(4)}=0.13$. The collective IRPR is:

$$
U R^{\mathbf{c}}=\left(\begin{array}{cccc}
\langle 0.50,0.50\rangle & \langle 0.37,0.43\rangle & \langle 0.36,0.37\rangle & \langle 0.29,0.28\rangle \\
\langle 0.43,0.37\rangle & \langle 0.50,0.50\rangle & \langle 0.46,0.43\rangle & \langle 0.40,0.34\rangle \\
\langle 0.37,0.36\rangle & \langle 0.43,0.46\rangle & \langle 0.50,0.50\rangle & \langle 0.41,0.40\rangle \\
\langle 0.28,0.29\rangle & \langle 0.34,0.40\rangle & \langle 0.40,0.41\rangle & \langle 0.50,0.50\rangle
\end{array}\right)
$$

Step 3. Computing proximate indexes: Using the Hamming distance, we have:

Level 1. The proximity indexes on the pair of alternatives for each expert are:

$$
\begin{aligned}
& \left(P P_{i j}^{1}\right)=\left(\begin{array}{llll}
1.00 & 0.91 & 0.83 & 0.85 \\
0.91 & 1.00 & 0.87 & 0.88 \\
0.83 & 0.87 & 1.00 & 0.89 \\
0.85 & 0.88 & 0.89 & 1.00
\end{array}\right) \quad\left(P P_{i j}^{2}\right)=\left(\begin{array}{llll}
1.00 & 0.94 & 0.90 & 0.86 \\
0.94 & 1.00 & 0.96 & 0.84 \\
0.90 & 0.96 & 1.00 & 0.84 \\
0.86 & 0.84 & 0.84 & 1.00
\end{array}\right) \\
& \left(P P_{i j}^{3}\right)=\left(\begin{array}{llll}
1.00 & 0.84 & 0.61 & 0.86 \\
0.84 & 1.00 & 0.73 & 0.83 \\
0.61 & 0.73 & 1.00 & 0.74 \\
0.86 & 0.83 & 0.74 & 1.00
\end{array}\right) \quad\left(P P_{i j}^{4}\right)=\left(\begin{array}{llll}
1.00 & 0.89 & 0.87 & 0.83 \\
0.89 & 1.00 & 0.93 & 0.86 \\
0.87 & 0.93 & 1.00 & 0.91 \\
0.83 & 0.86 & 0.91 & 1.00
\end{array}\right)
\end{aligned}
$$


Level 2. The proximity indexes on the alternatives for each expert are:

$$
\begin{aligned}
& \left(P A_{i}^{1}\right)=(0.863,0.887,0.863,0.873) ; \quad\left(P A_{i}^{2}\right)=(0.900,0.913,0.913,0.847) \\
& \left(P A_{i}^{3}\right)=(0.770,0.800,0.693,0.810) ; \quad\left(P A_{i}^{4}\right)=(0.863,0.893,0.903,0.867) .
\end{aligned}
$$

Level 3. The proximity indexes on the relation for each expert are:

$$
P I^{1}=0.870, P I^{2}=0.890, P I^{3}=0.768, P I^{4}=0.883 .
$$

Step 4. Computing consensus levels: For illustration purposes, we are assuming a value of $\delta=0.68$, which in practice means that a greater weight is assigned to the consistency criterion than to the proximity criterion in the computation of consensus levels. This could well happen when experts would like to obtain a solution of consensus while maintaining a high level of consistency.

Level 1. The consensus levels of pair of alternatives are:

$$
\begin{aligned}
& \left(C L_{i j}^{1}\right)=\left(\begin{array}{llll}
1.000 & 0.958 & 0.929 & 0.938 \\
0.958 & 1.000 & 0.935 & 0.938 \\
0.929 & 0.935 & 1.000 & 0.951 \\
0.938 & 0.938 & 0.951 & 1.000
\end{array}\right) \quad\left(C L_{i j}^{2}\right)=\left(\begin{array}{llll}
1.000 & 0.964 & 0.927 & 0.931 \\
0.964 & 1.000 & 0.929 & 0.922 \\
0.927 & 0.929 & 1.000 & 0.925 \\
0.931 & 0.922 & 0.925 & 1.000
\end{array}\right) \\
& \left(C L_{i j}^{3}\right)=\left(\begin{array}{llll}
1.000 & 0.911 & 0.855 & 0.904 \\
0.911 & 1.000 & 0.886 & 0.895 \\
0.855 & 0.886 & 1.000 & 0.910 \\
0.904 & 0.895 & 0.910 & 1.000
\end{array}\right) \quad\left(C L_{i j}^{4}\right)=\left(\begin{array}{llll}
1.000 & 0.910 & 0.941 & 0.932 \\
0.910 & 1.000 & 0.967 & 0.925 \\
0.941 & 0.967 & 1.000 & 0.968 \\
0.932 & 0.925 & 0.968 & 1.000
\end{array}\right)
\end{aligned}
$$

Level 2. The consensus levels of alternatives are:

$$
\begin{array}{ll}
\left(C L_{i}^{1}\right)=(0.942,0.943,0.938,0.943) ; & \left(C L_{i}^{2}\right)=(0.941,0.938,0.927,0.926) \\
\left(C L_{i}^{3}\right)=(0.890,0.898,0.884,0.903) ; & \left(C L_{i}^{4}\right)=(0.928,0.934,0.959,0.942) .
\end{array}
$$

Level 3. The individual consensus levels are: $C L^{1}=0.941, C L^{2}=0.933, C L^{3}=0.896, C L^{4}=0.941$.

Setting the minimum consensus threshold value $\gamma$ at 0.9 , the feedback mechanism must be activated to assist expert $e_{3}$ because $C L^{3}=0.896<\gamma$.

Step 5. Feedback mechanism: The following APS set is obtained:

$$
A P S=\{(3,1,3),(3,3,1),(3,2,3),(3,3,2),(3,2,4),(3,4,2)\}
$$

The recommendations for expert $e_{3}$ are:

- Your missing preference value for the pair of alternatives $(1,3)$ should be as close as possible to $\langle 0.52,0,27\rangle$. 
- Your missing preference value for the pair of alternatives $(3,1)$ should be as close as possible to $\langle 0.27,0.52\rangle$.

- You should change your preference value for the pair of alternatives $(2,3),\langle 0.60,0.30\rangle$ to a value closer to $\langle 0.56,0.34\rangle$.

- You should change your preference value for the pair of alternatives $(3,2),\langle 0.30,0.60\rangle$ to a value closer to $\langle 0.34,0.56\rangle$.

- You should change your preference value for the pair of alternatives $(3,4),\langle 0.40,0.55\rangle$ to a value closer to $\langle 0.37,0.54\rangle$.

- You should change your preference value for the pair of alternatives $(4,3),\langle 0.55,0.30\rangle$ to a value closer to $\langle 0.54,0.37\rangle$.

Once experts implement the changes in their IRPRs, a new consensus process round takes place.

Second Consensus Round. Assuming the experts implement the values recommended above, the new collective IRPR would be

$$
U R^{\mathbf{c}}=\left(\begin{array}{cccc}
\langle 0.500,0.500\rangle & \langle 0.363,0.429\rangle & \langle 0.343,0.375\rangle & \langle 0.283,0.278\rangle \\
\langle 0.429,0.363\rangle & \langle 0.500,0.500\rangle & \langle 0.450,0.442\rangle & \langle 0.395,0.337\rangle \\
\langle 0.375,0.343\rangle & \langle 0.442,0.450\rangle & \langle 0.500,0.500\rangle & \langle 0.417,0.392\rangle \\
\langle 0.278,0.283\rangle & \langle 0.337,0.395\rangle & \langle 0.392,0.417\rangle & \langle 0.500,0.500\rangle
\end{array}\right)
$$

and the new consensus levels would become: $C L^{1}=0.943, C L^{2}=0.931, C L^{3}=0.905, C L^{4}=0.939$, which are all above the threshold value $\gamma=0.9$, and therefore the selection process would be activated.

Step 6. Selection process: The SRPR associated of the above IRPR $U R^{c}$ is

$$
\mathbf{P}=\left(\begin{array}{llll}
0.500 & 0.467 & 0.484 & 0.503 \\
0.533 & 0.500 & 0.504 & 0.529 \\
0.516 & 0.496 & 0.500 & 0.513 \\
0.497 & 0.471 & 0.487 & 0.500
\end{array}\right)
$$

Thus, we have:

$$
\mathbf{1}-\mathbf{P}^{\mathbf{s}}=\left(\begin{array}{cccc}
- & 0.934 & 0.968 & 1 \\
1 & - & 1 & 1 \\
1 & 0.992 & - & 1 \\
0.994 & 0.942 & 0.974 & -
\end{array}\right)
$$

Using the BUM function $Q=r^{1 / 2}$ to implement the linguistic majority ' $m o s t$ of', we obtain the OWA operator weighting vector $\omega=(0.58,0.24,0.18)^{T}$, and then compute the following intuitionistic quantifier guided non-dominance degree associated to each one of the alternatives:

$$
I Q G N D D_{1}=0.98044, I Q G N D D_{2}=1, I Q G N D D_{3}=0.99856, I Q G N D D_{4}=0.97984
$$


The alternatives can be ranked from best to worst according to the degree up to which an alternative is not dominated by 'most of' the rest of alternatives as follows:

$$
x_{2} \succ x_{3} \succ x_{1} \succ x_{4},
$$

Therefore, $x_{2}$ is the most important criteria.

\section{Conclusion}

'DEMOCRATIC THEORY is based on the premise that the resolution of a matter of social policy, group choice or collective action should be based on the desires or preferences of the individuals in the society, group or collective.' This quotation from Fishburn [21, page 3] fully justifies the use of preferences in group decision making. However, democracy is recognised when decisions are made applying majority voting rules, which are easy to understand when each vote counts the same. However, there are many practical situations when this is not the case specially when experts are allowed to indicate their degree of preference, which might be the case for example when selecting candidates for a job at a company. In these cases, it is necessary to apply a new type of majority rules which allow to calibrate the amount of support required for the winner alternative by means of a difference of intensity of preference, which in this paper has been modelled with the non-dominance degree, as well as in consensus and consistency criteria. Furthermore, there may be some real-life decision making cases where a decision maker (DM) may not be able to accurately express his/her preferences for some or all of the alternatives because he/she is not completely confident or presents some hesitation, making they use of intuitionistic fuzzy values very suitable to model and represent the DM's preference rather than other type of preference representation formats. Indeed, the use of intuitionistic fuzzy sets has been widely applied in multiple attribute decision making [49], industry meteorological service selection [24] and group decision making [44], to cite a few fields.

In this paper, a novel consensus model for GDM problems with incomplete IRPRs has been presented, which has the following main advantages with respect to other consensus models proposed in the literature:

1. It allows the presence of incomplete IRPRs, an issue that has not been successfully addressed by any previous consensus model. It is suitable to deal with decision making cases in which an expert may not be able to express his/her preferences for alternatives due to a lack of in-depth knowledge or time pressure.

2. It is based on a correct mathematical formulation and modelling of the multiplicative transitivity property of IRPR as it is derived using the correct generalisation methodology from a crisp context to a fuzzy context via Zadeh's Extension Principle, as opposed to the incorrect modelling of this property previously proposed by $\mathrm{Xu}$ in [44]. As a consequence, the correct derivation of the multiplicative consistency property of IRPR is achieved. 
3. It investigates a MC-IOWA operator to aggregate individual preference relations, which assures a monotonic increasing mapping between the experts' consistency levels and their contribution weight in the collective IRPR, and ultimately in the solution of the GDM problem.

4. It builds consensus by incorporating both the consistency index (CI) and the proximity index (PI), and therefore consensus is assured to be achieved with a high level of consistency. The CI is computed using the derived based on the multiplicative transitivity property of preferences, which has been proved to be more appropriate to model consistency of preferences than the additive transitivity property [27-29, 34].

5. It proposes a novel feedback process that implements visual representation (Fig. 3(b)) to help experts to easily 'see' their relative consensus position within the group and to identify the experts contributing less to consensus. Additionally, visual simulation of future consensus status if the recommended values were to be implemented are also implemented within the feedback process. In the light of this visual extra information, experts can revisit their evaluations and make changes if considered appropriate to achieve a higher consensus level. Therefore, our proposed feedback mechanism differs with respect to other feedback mechanisms that force experts to change their opinions in the literature $[29,40]$. Also, it need less computation burden than the feedback mechanisms in [29] because it takes the reciprocity property into account in its design, and it does not need to re-estimate values already estimated in previous steps of the consensus model.

6. Finally, the feedback mechanism is designed following a top to bottom methodology, and therefore it focuses exclusively on identifying those experts that are furthest from the group and consequently contributing less to consensus. Once these experts are identified, the model proceeds to identify their alternatives and the corresponding preference values, at the level of pair of alternatives, that are more in conflict in terms of consensus with the corresponding group values. Having reached this lowest level, the feedback mechanism also provides specific values to the experts to guide them in the direction that would make them increase their contribution to consensus, while preserving their internal consistency, and ultimately to contribute positively in obtaining a group solution of consensus.

The proposed consensus process still exhibits some limitations to be addressed in future research.

1. The MC-IOWA operator aggregates experts's IRPRs taking into account only the experts's consistency levels. Other factors influencing experts' weights, such as knowledge, experience and reputation of experts [53], are not considered because they are difficult to determine directly. A potential avenue to explore in future to address this issue is the implementation of trust network 
analysis to capture and measure trust among the sets of experts and derive trust-consistency based experts' weights.

2. In previously developed GDM models [27-29, 34], the threshold value of consensus $\gamma$ is provided beforehand. Since this threshold value affects the numbers of feedback rounds, it is interesting to investigate how the threshold value of consensus is determined by the group of experts in real scenarios and in particular to analyse the factors affecting its choosing.

\section{Acknowledgements}

The authors are very grateful to the anonymous referees for their valuable comments and suggestions that have helped us to improve considerably the quality of this paper. This work was supported by National Natural Science Foundation of China (NSFC)under the Grant No.71101131 and No. 71331002, and Zhejiang Provincial National Science Foundation for Distinguished Young Scholars of China (No. LR13G010001).

[1] Alonso, S., Chiclana, F., Herrera, F., and Herrera-Viedma, E. (2004). A learning procedure to estimate missing values in fuzzy preference relations based on additive consistency. Lecture Notes Artificial Intelligence, 3131, 227-238.

[2] Alonso, S., Chiclana, F., Herrera, F., Herrera-Viedma, E., Alcala-Fdez, J., and Porcel, C. (2008). A consistency-based procedure to estimate missing pairwise preference values. International Journal of Intelligent Systems 23, 155-175.

[3] Atanassov, K. T. (1986). Intuitionistic fuzzy sets. Fuzzy Sets and Systems 20(1), 87-96.

[4] Atanassov, K. T. (2012). On intuitionistic fuzzy sets theory. Studies in Fuzziness and Soft Computing 283, Springer-Verlag Berlin Heidelberg.

[5] Bezdek, J. C., Spillman, B., and Spillman, R. (1978). A fuzzy relation space for group decision theory. Fuzzy Sets and systems 1(4), 255-268.

[6] Fujita, H., Hakura, J., and Kurematsu, M. (2009) Intelligent human interface based on mental cloning-based software. Knowledge-Based Systems 22(3), 216-234.

[7] Fujita, H., Hakura, J., and Kurematsu, M. (2010) Virtual Doctor System (VDS): Medical Decision Reasoning based on Physical and Mental ontologies. The 23rd International Conference on Industrial, Engineering \& Other Applications of Applied Intelligent Systems (IEA-AIE 2010), Springer, Vol.6097.

[8] Fujita, H., Hakura, J., and Kurematsu, M. (2010) Mental cloning base virtual diagnostician system: Virtual Medical Doctor(VMD) reasoning system. Proceedings of Third International Conference on Health Informatics, 250-256. 
[9] Fujita, H., Hakura, J., and Kurematsu, M. (2010) Virtual Doctor System (VDS): Framework on reasoning issues. Frontiers in Artificial Intelligence and Applications, Vol. 217. New Trends in Software Methodologies, Tools and Techniques - Proceedings of the 9th SoMeT-10, 481-489.

[10] Chiclana, F., Herrera, F., and Herrera-Viedma, E. (1998). Integrating three representation models in fuzzy multipurpose decision making based on fuzzy preference relations. Fuzzy Sets and Systems 97(1), 33-48.

[11] Chiclana, F., Herrera-Viedma, E., Herrera, F., and Alonso, S. (2007). Some induced ordered weighted averaging operators and their use for solving group decision-making problems based on fuzzy preference relations. European Journal of Operational Research 182, 383-399.

[12] Chiclana, F., Herrera-Viedma, E., Alonso, S., and Herrera, F. (2009). Cardinal consistency of reciprocal preference relations: a characterization of multiplicative transitivity. IEEE Transactions on Fuzzy Systems 17(1), 14-23.

[13] Chiclana, F., Mata, F., Alonso, S., Herrera-Viedma, E. and Martínez, L. (2008). Integration of a consistency control module within a consensus decision making model. International Journal of Uncertainty, Fuzziness and Knowledge Based Systems, 16, 35-53.

[14] Chiclana, F., Herrera-Viedma, E., and Alonso, S. (2007). A note on two methods for estimating missing pairwise preference values. IEEE Transactions on Systems, Man, and Cybernetics, Part B: Cybernetics 39(6), 1628-1633.

[15] Chiclana, F., Tapia-Garcia, J. M., del Moral, M. J., and Herrera-Viedma, E. (2013). A statistical comparative study of different similarity measures of consensus in group decision making. Information Sciences, 221, 110-123.

[16] Cutello, V., and Montero, J. (1994). Fuzzy rationality measures. Fuzzy Sets and Systems, 62, $39-54$.

[17] Deschrijver, G., and Kerre, E. E. (2003). On the relationship between some extensions of fuzzy set theory. Fuzzy Sets and Systems 133(2), 227-235.

[18] Dong, Y. C., Zhang, G. Q., Hong, W. C.,and Xu, Y. F. (2010). Consensus models for AHP group decision making under row geometric mean prioritization method. Decision Support Systems $49(3), 281-289$.

[19] Dong, Y. C., Li, C. C., Xu, Y. F., and Gu, X. (In press). Consensus-based group decision making under multi-granular unbalanced 2-tuple linguistic preference relations. Group Decision and Negotiation DOI 10.1007/s10726-014-9387-5 
[20] Fedrizzi, M., and Giove, S. (2007). Incomplete pairwise comparison and consistency optimization. European Journal of Operational Research 183, 303-313.

[21] Fishburn, P.C. (1973). The theory of social choice. Princeton University Press, Princeton.

[22] Fodor, J., Roubens, M., (1994b). Fuzzy preference modelling and multicriteria decision support. Kluwer Academic Publishers.

[23] Gong, Z. W., Li, L. S., Zhou, F. X., and Yao, T. X. (2009). Goal programming approaches to obtain the priority vectors from the intuitionistic fuzzy preference relations. Computers and Industrial Engineering 57, 1187-1193.

[24] Gong Z. W., Li, L. S., Jeffrey, F., and Zhao, Y. (2011). The optimal priority models of the intuitionistic fuzzy preference relation and their application in selecting industries with higher meteorological sensitivity. Expert Systems with Applications 38, 4394-4402.

[25] Hanss, M. (2005). Applied Fuzzy Arithmetic. An Introduction with Engineering Applications. Springer-Verlag Berlin Heidelberg.

[26] Herrera F., Alonso S., Chiclana F., and Herrera-Viedma E. (2009). Computing with words in decision making: foundations, trends and prospects. Fuzzy Optimization and Decision Making $8(4), 337-364$.

[27] Herrera-Viedma, E., Herrera, F., Chiclana, F., and Luque, M. (2004). Some issues on consistency of fuzzy preference relations. European journal of operational research 154(1), 98-109.

[28] Herrera-Viedma, E., Chiclana, F., Herrera, F., and Alonso, S. (2007). Group decision-making model with incomplete fuzzy preference relations based on additive consistency. IEEE Transactions on Systems, Man, and Cybernetics, Part B: Cybernetics 37(1), 176-189.

[29] Herrera-Viedma, E., Alonso, S., Chiclana, F., and Herrera, F.(2007). A consensus model for group decision making with incomplete fuzzy preference relations. IEEE Transactions on Fuzzy Systems 15(5), 863-877.

[30] Luce, R. D., and Suppes, P. (1965). Preferences, utility and subject probability. In: Handbook of Mathematical Psychology, Vol. III (eds. R.D. Luce et al.), Wiley, New York, 249-410.

[31] Ovchinnikov, S., and Roubens, M. (1992). On fuzzy stric preference, indifference, and incomparability relations. Fuzzy Sets Systems 49(1), 15-20.

[32] Pérez-Asurmendi, P., and Chiclana, F. (2014) Linguistic majorities with difference in support. Applied Soft Computing 18, 196 - 208. 
[33] Perny, P., and Roubens, M. (2010). Fuzzy preference modelling. In Slowinski, R. (ed.) (1998). Fuzzy Sets in Decision Analysis, Operations Research and Statistics. The Handbooks of Fuzzy Sets Series, 1, 3-30.

[34] Szmidt, E., and Kacprzyk, J. (2003). A consensus-reaching process under intuitionistic fuzzy preference relations. International Journal Intelligent System 18, 837-852.

[35] Saaty, T. L. (1980). The analytic hierarchy process. New York: McGraw-Hill.

[36] Tanino, T. (1984). Fuzzy preference orderings in group decision making. Fuzzy sets and system 12, $117-131$.

[37] Wu, J., Li, J. C., Li, H., and Duan, W. Q. (2009). The induced continuous ordered weighted geometric operators and their application in group decision making. Computers and Industrial Engineering 57, 1545-1552.

[38] Wu, J., Cao, Q. W., and Zhang, J. L. (2010). Some properties of the induced continuous ordered weighted geometric operators in group decision making. Computers and Industrial Engineering 59, 100-106.

[39] Wu, J., and Chiclana, F. (2012). Non-dominance and attitudinal prioritisation methods for intuitionistic and interval-valued intuitionistic fuzzy preference relations. Expert Systems with Applications 39, 13409-13416.

[40] Wu, J., and Chiclana, F. (2014). A social network analysis trust-consensus based approach to group decision-making problems with interval-valued fuzzy reciprocal preference relations. Knowledge-Based Systems 99, 97-107.

[41] Wu, J., and Chiclana, F. (2014). Visual information feedback mechanism and attitudinal prioritisation method for group decision making with triangular fuzzy complementary preference relations. Information Sciences 279, 716-736.

[42] Wu, Z. B., and Xu J. P. (2012) A consistency and consensus based decision support model for group decision making with multiplicative preference relations. Decision Support Systems 52, $757-767$.

[43] Xu, Z. S. (2007a). A survey of preference relations. International Journal of General System $27(36), 179-203$.

[44] Xu, Z. S. (2007b). Intuitionistic preference relations and their application in group decision making. Information Sciences 177, 2363-2379. 
[45] Yager, R. R. (1996). Quantifier guided aggregation using OWA operators. International Journal of Intelligent Systems, 11, 49-73.

[46] Yager, R. R., and Filev, D. P. (1999). Induced ordered weighted averaging operators. IEEE Transactions on Systems, Man and Cybernetics 29, 141-150.

[47] Yager, R. R., and Rybalov, A. (1996). Uninorms aggretation operators. Fuzzy Sets and Systems 80,111-120.

[48] Yang, Y., and Chiclana, F. (2012). Consistency of 2d and 3d distances of intuitionistic fuzzy sets. Expert Systems with Applications 39(10), 8665-8670.

[49] Ye, J. (2010). Fuzzy decision-making method based on the weighted correlation coefficient under intuitionistic fuzzy environment. European Journal of Operational Research 205(1), 202-204.

[50] Yu, L. A., and Lai, K. K. (2011). A distance-based group decision-making methodology for multi-person multi-criteria emergency decision support. Decision Support Systems 51, 307-315.

[51] Zadeh, L. A. (1965). Fuzzy sets. Information and Control 8 (3), 338-357.

[52] Zadeh, L. A. (1975). The concept of a linguistic variable and its application to approximate reasoning-I. Information Sciences 8, 199-249.

[53] Zhang, F., Ignatius, J., Lim, Ch. P. and Zhang, Y. (2014). A hybrid weighted aggregation method based on consistency and consensus in group decision making. Proceedings of the 2014 IEEE International Conference on Fuzzy Systems, Beijing, China. 